\title{
Identification of Suitable Agents against Adenine Phosphoribosyl Transferase for the Management of Leishmaniasis: Synthesis, Characterization and Computational Studies
}

\author{
Ravinder Sharma ${ }^{1}$ (D), Divya Yadav ${ }^{1}\left(\mathbb{D}\right.$, Rakesh Yadav $^{2, *(\mathbb{D})}$ \\ Department of Pharmacy, Banasthali Vidyapith, Banasthali-304022, Rajasthan, India \\ Amity Institute of Pharmacy, Amity University, Manesar-122413, Gurugram, Haryana, India \\ * Correspondence: rakesh_pu@yahoo.co.in
}

Scopus Author ID 57189023768

Received: 8.10.2021; Revised: 5.11.2021; Accepted: 8.11.2021; Published: 25.11.2021

\begin{abstract}
A leishmaniasis is a group of diseases attributable to protozoan parasites of the genus Leishmania. It is a potential disease mostly occurring in developing nations. Various quinoline substituted derivatives (11a-f, 12a-f, and 13a-f) were synthesized by refluxing amino quinolines with an equivalent number of different alkylaminoethyl chlorides and evaluated for their in vitro antileishmanial activity against promastigotes forms of Leishmania donovani by using MTT [3(4,5-dimethylthiazol-2-yl)-2,5-diphenyl tetrazolium bromide] reduction assay. Compounds $\mathbf{1 1 f}\left(\mathrm{IC}_{50}=\right.$ $13.61 \mu \mathrm{g} / \mathrm{mL}), 12 \mathrm{f}\left(\mathrm{IC}_{50}=11.92 \mu \mathrm{g} / \mathrm{mL}\right)$ and $\mathbf{1 3 f}\left(\mathrm{IC}_{50}=10.41 \mu \mathrm{g} / \mathrm{mL}\right)$ have shown significant antileishmanial activity when compared with standard sitamaquine $\left(\mathrm{IC}_{50}=10.09 \mu \mathrm{g} / \mathrm{mL}\right)$. Furthermore, the molecular docking analysis targeting adenine phosphoribosyltransferase of Leishmania donovani exhibits significant binding interactions. In silico, ADMET predictions revealed that these compounds, i.e., 11f, 12f, and 13f, demonstrated good absorption as well as solubility characteristics with good druglikeness and drug score values compared to the standard drug.
\end{abstract}

Keywords: antileishmanial activity; Leishmania donovani; molecular docking study; ADMET properties; drug-likeness.

(C) 2021 by the authors. This article is an open-access article distributed under the terms and conditions of the Creative Commons Attribution (CC BY) license (https://creativecommons.org/licenses/by/4.0/).

\section{Introduction}

Leishmaniasis is among the most prevalent parasitic disease caused by unicellular flagellate intracellular protozoa. The biological classification of microbe indicates that it belongs to the genus Leishmania [1,2]. The disease mostly develops in deprived and developing nations. The Indian subcontinent and the African continent are most prone to the disease $[3,4]$. Leishmaniasis can be caused by more than 20 species of Leishmania. It may produce different clinical symptoms, which may vary from self-healing cutaneous ulcers to severe visceral diseases. The transmission mode may be from humans to humans, i.e., anthroponotic, or via a vector from an animal reservoir, i.e., zoonotic [5]. Depending upon the syndromes of leishmaniasis, it can be categorized as visceral leishmaniasis, cutaneous leishmaniasis, mucosal leishmaniasis, and post-kala-azar leishmaniasis [6]. Today approximately one billion people live in the endemic areas, and about 30,000 new cases of visceral leishmaniasis and more than a million cases of cutaneous leishmaniasis are reported annually [7,8]. All patients diagnosed with leishmaniasis must be treated properly, or the results may be fatal [9]. Figure 1 depicts the important drugs which are currently being used for curing leishmaniasis [10]. But due to the complexity of the causative organism, the treatment options for leishmaniasis need to be diversified [11]. 

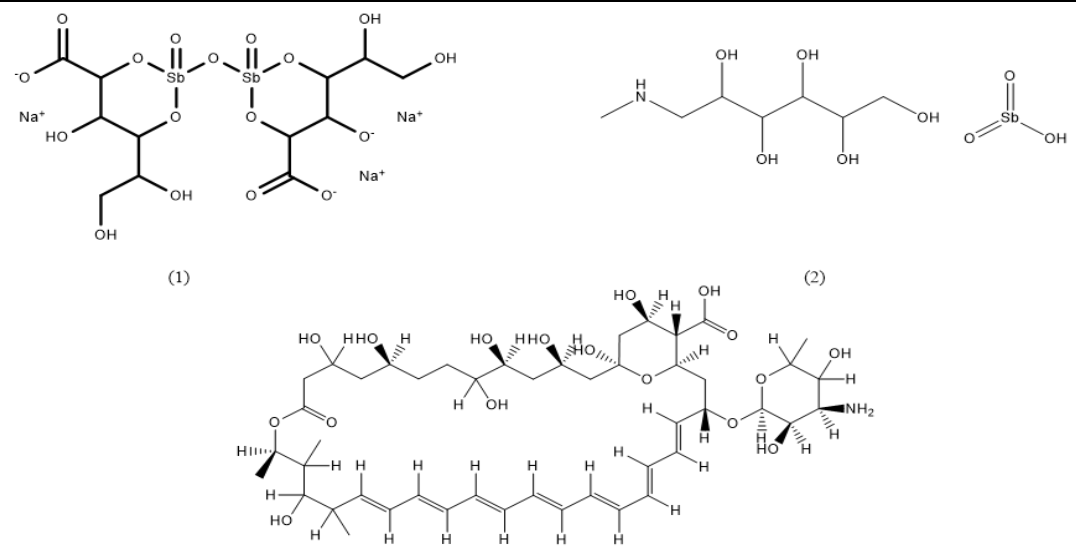

(3)
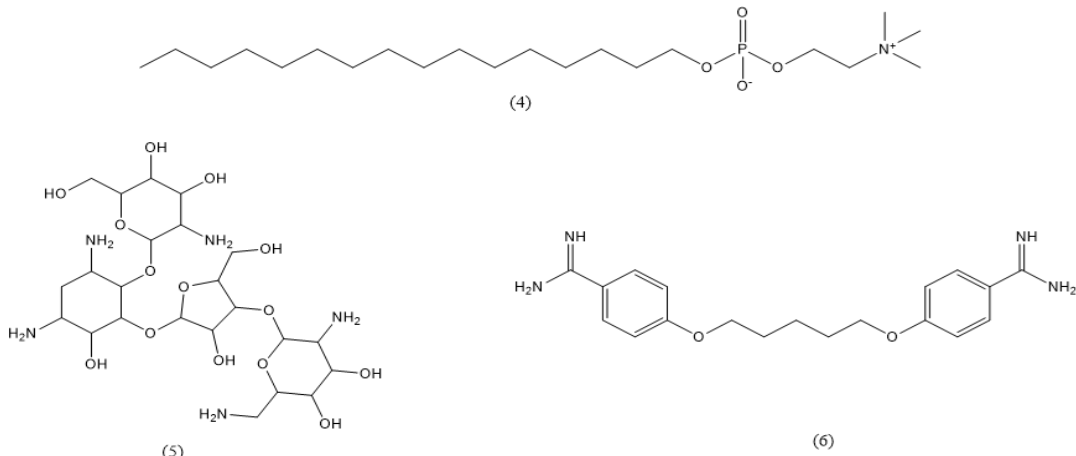

(5)

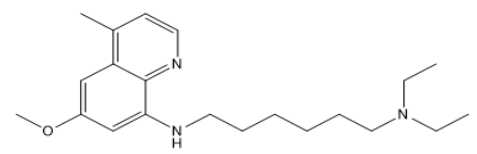

Figure 1. Clinically employed drugs for the treatment of leishmaniasis. (1) Sodium stibogluconate, (2) Meglumine antimoniate, (3) Amphotericin B, (4) Miltefosine, (5) Paromomycin, (6) Pentamidine, (7)

Sitamaquine.

Paromomycin is an antibiotic of the aminoglycoside category and is mostly a secondline drug for the treatment of leishmaniasis. It is commonly used for intestinal infections. It acts by inhibiting the synthesis of proteins by binding to $16 \mathrm{~S}$ rRNA, causing the incorrect incorporation of amino acids in the nascent peptide chain [10, 12].

Pentavalent antimonials are the first-line remedies for treating leishmaniasis. The class includes drugs like sodium stibogluconate and meglumine antimoniate [13]. They primarily act by inhibiting the relaxation of the supercoiled plasmid pBR322 catalyzed by DNA topoisomerase of the microbe and also induce changes in the parasite cell membrane [14].

Amphotericin B isolated from Streptomyces nodosus has replaced pentavalent antimonials as the first-line drugs for treating leishmaniasis as they have about $\sim 97 \%$ cure rate [15]. The polyene antibiotic of this class, i.e., Liposomal Amb is the most effective that attacks the cell wall of the parasite, causing the leakage of metabolites from the parasite cell, thus causing the death of the parasite [16,17].

Miltefosine is the only drug that is taken orally to treat leishmaniasis. It hinders the synthesis of phosphatidylcholine and also affects the mitochondria of the parasite. It is also observed that it disrupts intracellular $\mathrm{Ca}^{2+}$ homeostasis [18]. The current treatment options for leishmaniasis have great success rates (like Amphotericin B 97\%), but still, there are some problems with their usage. Amphotericin B presents many side effects like hypokalemia, immediate shocks upon infusion, which necessitates the close monitoring of the patient [19]. Pentavalent antimonials are still useful but are potentially toxic, and moreover, it requires 
painful injections for 20 days, which is also the case for Paromomycin whereas Miltefosine, shows the problem of being teratogenic [20]. Therefore, the buildout of unprecedented, innocuous, and inexpensive compounds having effective leishmanicidal activity is urgently desired.

Quinoline ring scaffold, which is considered to have efficacy against the Leishmania species, is one of the widely used literature frameworks for synthesizing novel derivatives with promising antiparasitic activity [21-28]. So, in the current line of work, we hereby proclaim the synthesis and characterization of 3,6, and 8-aminoquinoline derivatives as anti-leishmanial agents. Docking analysis of the various synthetic derivatives has been conducted to assess the best in silico confirmation, and amid them, the compounds having comparable binding affinity are further ascertained for antileishmanial activity against Leishmania donovani.

\section{Materials and Methods}

\subsection{General procedure for the synthesis of target molecules.}

2.1.1. Synthesis of substituted $\mathrm{N}$-quinoline amine derivatives.

Substituted 3, 6, or 8-amino quinolines derivatives were prepared by refluxing 3,6, or 8 -amino quinolines, i.e., (8), (9), or (10) with an equivalent amount of hydrochlorides of various alkylaminoethyl chlorides such as 4-(2-chloroethyl)-morpholine, 1-(2-chloroethyl)piperidine, 2-chloro- $N, N$-dimethyl ethanamine, 1-(2-chloroethyl)-pyrrolidine, 2-chloro- $N, N$ diethyl ethanamine, and 2-(2-chloroethyl)-isoindoline-1,3-dione in the presence of potassium carbonate at $80^{\circ} \mathrm{C}$ for $4-6 \mathrm{~h}$ using ethyl methyl ketone as a solvent. The formation of various compounds was confirmed by various physicochemical properties and spectral analysis (Scheme 1).

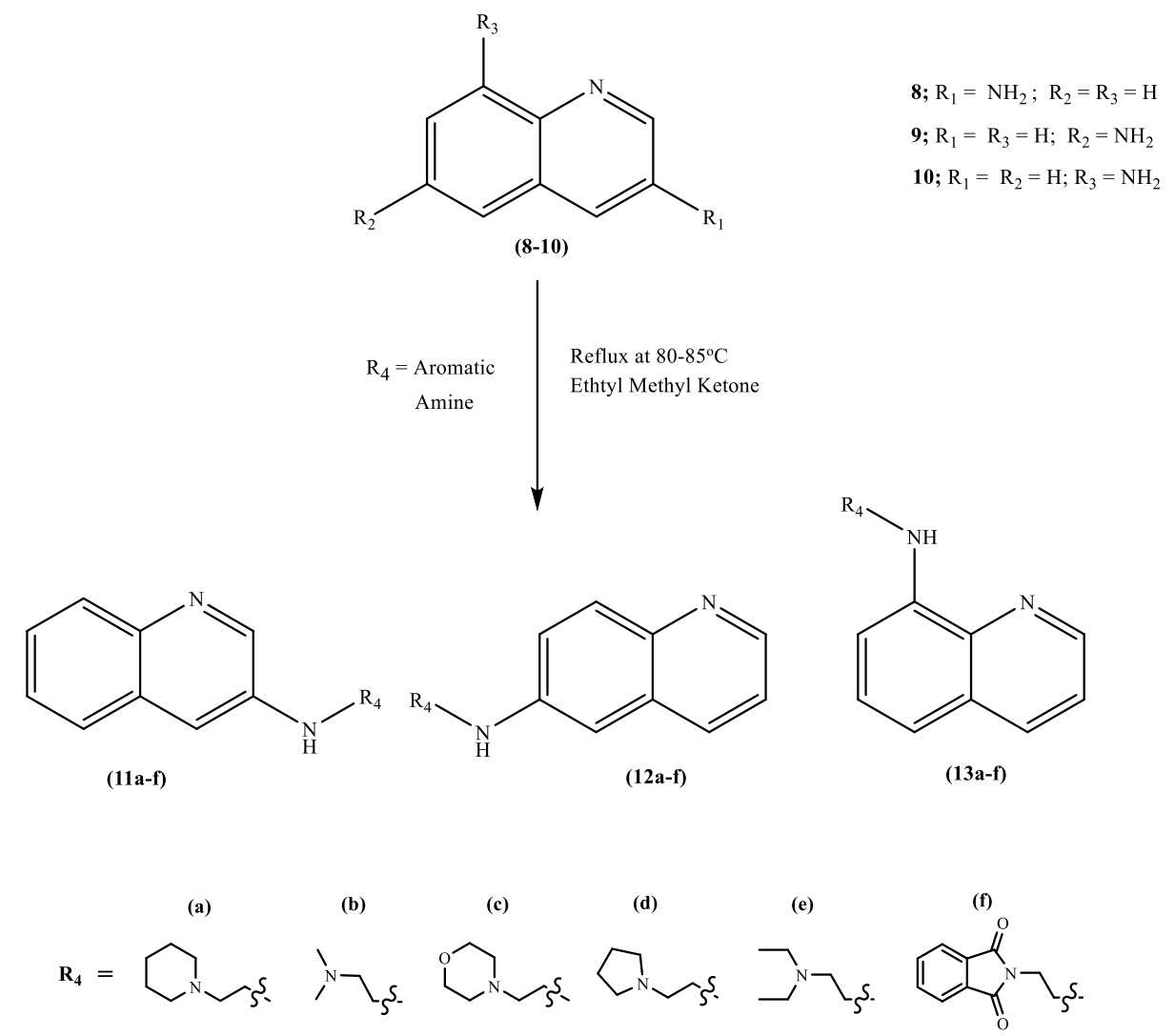

Scheme 1. Synthesis of various substituted quinoline derivatives, i.e., 11a-f, 12a-f, and 13a-f. 
2.2. Biological evaluation.

For preparing the stock solutions, i.e., $(10 \mathrm{mg} / \mathrm{mL})$ of the synthetic quinoline derivatives, about $10 \mathrm{mg}$ of the sample compounds is dissolved in $1 \mathrm{ml}$ of DMSO. To get required dilutions varying from $2.5 \mu \mathrm{g} / \mathrm{mL}$ to $100 \mu \mathrm{g} / \mathrm{mL}$ each stock solution was further diluted with Roswell Park Memorial Institute (RPMI) complete media. The influence of tested molecules upon the survivability of promastigote form of Leishmania donovani has been estimated by measuring the metabolism of MTT [3-(4,5-dimethylthiazol-2-yl)-2,5-diphenyltetrazolium bromide] following an incubation period of $96 \mathrm{~h}$. Cells were inoculated at $1 \times 106$ $/ 100 \mu \mathrm{L}$ RPMI-1640 in each cavity of 96-well flat-bottom microtiter plates. Further to accomplish preferred concentrations, $100 \mu \mathrm{L}$ of media per well with different concentrations of synthesized compounds $(2.5 \mu \mathrm{g} / \mathrm{ml}$ to $100 \mu \mathrm{g} / \mathrm{ml})$ or standard drug $(2.5 \mu \mathrm{g} / \mathrm{ml}$ to $50 \mu \mathrm{g} / \mathrm{ml})$, dissolved in DMSO $[29,30]$ were added in triplicate. The plates were incubated at $37{ }^{\circ} \mathrm{C}$ for a period of $72 \mathrm{~h}$ before the addition of MTT (10 $\mu \mathrm{L}$ per well of a $5 \mathrm{mg} \mathrm{mL}-1$ PBS stock), and then plates were incubated additionally for 4-5 hours in $\mathrm{CO}_{2}$ incubator. MTT processing was stopped and formazan crystals solubilized by adding $50 \mu \mathrm{L}$ of DMSO per well and incubated overnight at $37{ }^{\circ} \mathrm{C}$. The relative amount of formazan per well produced by viable cells was measured photometrically at $590 \mathrm{~nm}$. The experiments were performed in triplicate for the determination of the sensitivity of each compound.

\subsection{Computational studies.}

\subsubsection{Molecular docking.}

The molecular docking procedure was used to predict the binding interactions between the quinoline derivatives and the binding pocket of the enzyme adenine phosphoribosyltransferase from Leishmania donovani for further pharmacological evaluation. Binding energy $\left(\mathrm{kcal} \mathrm{mol}^{-1}\right)$ is the significant key parameter generated as an outcome of molecular docking, indicating the affinity and intensity of the interaction among the ligand and the receptor. The binding energy and intensity of interaction are reciprocal to each other, i.e., and the more is the binding energy, the less will be the intensity of the interaction and vice versa. Therefore, during docking studies, the ligand which displays the minimal binding energy is considered ideally suited for further evaluation.

\subsubsection{Preparation of target for docking analysis.}

The 3D crystal structure of the adenine phosphoribosyltransferase from Leishmania donovani [31] (PDB ID: 1QCD with a resolution of 2.48 ) was retrieved from the protein databank. After retrieving the structure, all the water and ions molecules were relinquished, and the hydrogen atoms were added to the protein by the protonation using the DS visualizer (version v20.1.0.19295) software [32]. The active site was determined using the site sphere method of Discovery visualizer. Energy minimization of protein was performed with DS using CHARMm(Chemistry at Harvard Macromolecular Mechanics) and MMFF94 (Merck molecular) force field.

\subsubsection{D Structure validation.}

To check the structural integrity, i.e., the stereochemical quality of the 3D model of protein 1QCD, PROCHECK [33,34], a program that relies on Ramachandran plots for structure verification, was used (Figure 2). Ramachandran Plot of the prepared protein represents $89.1 \%$ 
(172 amino acids) of the total residues in the most favored region and 10.7\% (21 amino acids) in the additionally allowed region, indicating a good quality model for study.

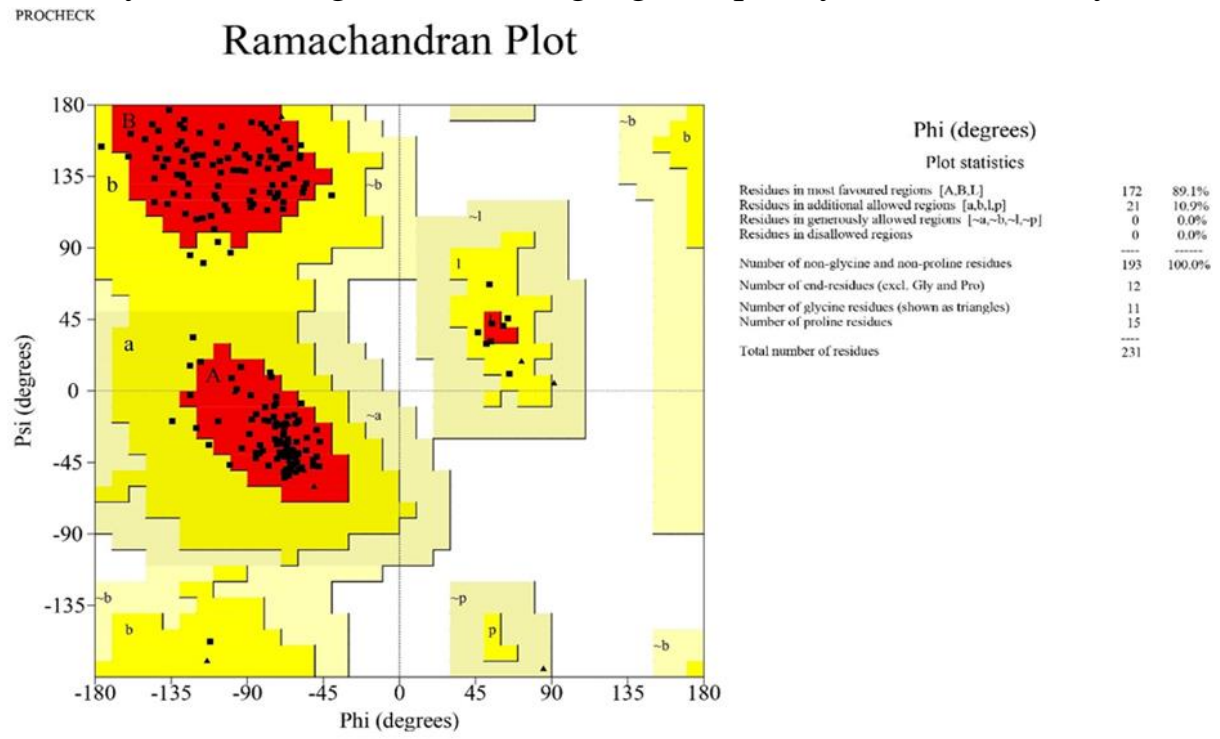

Figure 2. Stereochemical analysis of $1 \mathrm{QCD}$. The red region declares the most favorable area of residues; the yellow region is additionally allowed.

\subsubsection{Preparation of ligand for docking analysis.}

Each ligand structure was built by Chem3D pro version 12.0.2.1076 and optimized using MMFF94 force field and employing conjugate gradients optimization algorithm with around 200 number of steps in PYRX software. After energy minimizations, ligands are converted into PDBQT format using Open Bable GUI software embedded in PYRX [35].

\subsubsection{Docking methodology and analysis}

Computational docking is performed to get various probable conformations and orientations of the ligands with the binding site. The grid center for docking was set $\mathrm{X}=$ 28.9567, $Y=28.6635$, and $Z=7.588$ for $1 Q C D$. After this, virtual screening was carried by rigid molecular docking into the active site of the protein. Throughout the virtual screening, the ligand molecules were flexible, and the macromolecule was kept rigid. Finally, the result of binding energy was extracted from the software.

\subsection{Drug-likeness and ADMET evaluation.}

Inadequate pharmacokinetic and toxicity studies lead to failure of the drug development process in the final stages.

Therefore, in order to escalate the drug discovery process, primary assessment of pharmacokinetics and toxicity studies is the mandate step [36-38].

\subsubsection{Drug-likeness.}

Drug-likeness is an important aspect in drug development for the screening of drug molecules promptly. This parameter is being used as a process to correlate the physicochemical aspect of a drug molecule with its biopharmaceutical aspect in the human body, particularly its impact on bioavailability in the oral route [39]. Therefore, all the synthetic derivatives were assessed for their drug-like nature under Lipinski's rules of five by employing DruLiTo software [40]. As per Lipinski's rule, the Log P of "drug-like" molecules should be less than 5, 
molecular weight (M.W.) below 500, Total polar surface area under 140 and number of hydrogen bond acceptors (HBA), as well as the number of hydrogen bond donors (HBD) below 10 and 5 respectively [41].

\subsubsection{ADMET analysis.}

To assess different pharmacokinetic properties like absorption, distribution, metabolism, excretion, and toxicity of synthetic compounds within the human body ADMETlab web platform was used [42]. The ADMETlab envisaged the Human Intestinal Absorption (HIA+ or HIA-), Blood-Brain Barrier Penetration (BBB+ or BBB-), Caco-2 Permeability (permeable or non-permeable), Human Hepatotoxicity (H-HT), Solubility, i.e., LogS value, and Acute toxicity (LD50).

\section{Results}

\subsection{Spectral data.}

\section{$\mathrm{N}$-(2-(Piperidine-1-yl)-ethyl)-quinoline-3-amine (11a)}

Yield: 31\%, m.p:118-121 ${ }^{\circ} \mathrm{C}$, FT-IR (KBr, $\left.\mathrm{cm}^{-1}\right): 1105$ (C-N), 1450 (C=C), 1671 $(\mathrm{C}=\mathrm{N}), 3609(-\mathrm{NH}-) .{ }^{1} \mathrm{H}-\mathrm{NMR}\left(\mathrm{DMSO}-d_{6}\right): \delta 1.82\left(\mathrm{~m}, 6 \mathrm{H},\left(-\mathrm{CH}_{2}\right)_{3}\right.$, piperidine), $2.70(\mathrm{t}, 4 \mathrm{H},(-$ $\left.\left.\mathrm{NCH}_{2}\right)_{2}\right), 2.65\left(\mathrm{t}, 2 \mathrm{H},-\mathrm{CH}_{2}-\right), 3.30\left(\mathrm{~m}, 2 \mathrm{H},-\mathrm{CH}_{2}-\right), 4.80(\mathrm{t}, 1 \mathrm{H},-\mathrm{NH}), 7.15$ (s, 1H, CH, Ar), $7.63(\mathrm{~m}, 2 \mathrm{H},-\mathrm{CH}, \mathrm{Ar}) 7.83$ (d, 2H, CH, Ar) and 8.23 (s, 1H, CH, Ar) ppm. Elemental analysis $\left(\mathrm{C}_{16} \mathrm{H}_{21} \mathrm{~N}_{3}\right)$; Calcd. C, 75.26; H, 8.29; N, 16.46; found: $\mathrm{C}, 74.95 ; \mathrm{H}, 7.70 ; \mathrm{N}, 15.47$.

\section{$\mathrm{N}$-(2-(Dimethylamino)-ethyl)-quinoline-3-amine (11b)}

Yield: $42 \%$, m.p: $129-132{ }^{\circ} \mathrm{C}$, FT-IR $\left(\mathrm{KBr}, \mathrm{cm}^{-1}\right): 1133(\mathrm{C}-\mathrm{N}), 1341(\mathrm{C}-\mathrm{H}), 1516(\mathrm{C}=\mathrm{C})$, $1633(\mathrm{C}=\mathrm{N}), 1783\left(\mathrm{C}-\mathrm{H}\right.$, ring), $3340(\mathrm{NH}) .{ }^{1} \mathrm{H}-\mathrm{NMR}\left(\mathrm{DMSO}-d_{6}\right): \delta 2.32\left(\mathrm{~s}, 6 \mathrm{H},\left(-\mathrm{N}\left(\mathrm{CH}_{3}\right)_{2}\right)\right.$, 2.73 (t, 2H, - $\left.\mathrm{CH}_{2}-\right), 3.30$ (m, 1H, - $\left.\mathrm{CH}_{2}-\mathrm{N}-\right), 4.28(\mathrm{t}, 1 \mathrm{H},-\mathrm{NH}), 7.44$ (s, 1H, CH, Ar), 7.62 (m, $2 \mathrm{H},-\mathrm{CH}, \mathrm{Ar}), 7.83(\mathrm{~d}, 1 \mathrm{H}, \mathrm{CH}, \mathrm{Ar}), 8.11(\mathrm{~d}, 1 \mathrm{H}, \mathrm{CH}, \mathrm{Ar})$ and $8.25(\mathrm{~s}, 1 \mathrm{H}, \mathrm{CH}, \mathrm{Ar}) \mathrm{ppm}$. Elemental analysis $\left(\mathrm{C}_{13} \mathrm{H}_{17} \mathrm{~N}_{3}\right)$; Calcd. C, 72.52; H, 7.96; N, 19.52; found: C, 72.23; H, 7.40; N, 18.34.

\section{$\mathrm{N}$-(2-Morpholinoethyl)-quinoline-3-amine (11c)}

Yield: 33\%, m.p: 124-127 ${ }^{\circ} \mathrm{C}$, FT-IR (KBr, cm$\left.{ }^{-1}\right): 1089$ (C-O), $1271(\mathrm{C}-\mathrm{N}), 1595$ (C=C), $1670(\mathrm{C}=\mathrm{N}), 3661(-\mathrm{NH}-) .{ }^{1} \mathrm{H}-\mathrm{NMR}\left(\mathrm{CDCl}_{3}\right): \delta 2.16\left(\mathrm{t}, 4 \mathrm{H},\left(-\mathrm{NCH}_{2}\right)_{2}\right.$, morpholine), $2.05(\mathrm{t}$, $\left.2 \mathrm{H},-\mathrm{CH}_{2}-\right), 2.62\left(\mathrm{~m}, 2 \mathrm{H},-\mathrm{CH}_{2}-\right), 3.63\left(\mathrm{t}, 4 \mathrm{H},\left(-\mathrm{OCH}_{2}\right)_{2}\right.$, morpholine $), 4.18(\mathrm{t}, 1 \mathrm{H},-\mathrm{NH}), 7.05$ (s, 1H, CH, Ar), $7.53(\mathrm{~m}, 2 \mathrm{H},-\mathrm{CH}, \mathrm{Ar}), 7.80(\mathrm{~d}, 2 \mathrm{H}, \mathrm{CH}, \mathrm{Ar}) 8.27$ (s, 1H, CH, Ar) ppm. Elemental analysis $\left(\mathrm{C}_{15} \mathrm{H}_{19} \mathrm{~N}_{3}\right)$; Calcd. $\mathrm{C}, 70.01 ; \mathrm{H}, 7.44 ; \mathrm{N}, 16.33$; found: $\mathrm{C}, 69.73 ; \mathrm{H}, 6.91$; N, 15.35.

\section{$\mathrm{N}$-(2-(Pyrrolidine-1-yl)-ethyl)-quinoline-3-amine (11d)}

Yield: 39\%, m.p: $116-119{ }^{\circ} \mathrm{C}$, FT-IR $\left(\mathrm{KBr}, \mathrm{cm}^{-1}\right): 1191(\mathrm{C}-\mathrm{N}), 1474(\mathrm{C}-\mathrm{H}), 1520$ $(\mathrm{C}=\mathrm{C}), 1684(\mathrm{C}=\mathrm{N}), 1789(\mathrm{C}-\mathrm{H}), 3298(\mathrm{~N}-\mathrm{H}) .{ }^{1} \mathrm{H}-\mathrm{NMR}\left(\mathrm{DMSO}-d_{6}\right): \delta 1.72\left(\mathrm{~m}, 4 \mathrm{H},\left(-\mathrm{CH}_{2}-\right)_{2}\right.$, pyrrolidine), $2.75\left(\mathrm{t}, 2 \mathrm{H},-\mathrm{CH}_{2}-\right), 2.97\left(\mathrm{t}, 4 \mathrm{H},\left(-\mathrm{NCH}_{2}\right)_{2}\right.$, pyrrolidine), $3.31\left(\mathrm{~m}, 2 \mathrm{H},-\mathrm{CH}_{2}-\right), 4.23$ (t, 1H, -NH), 7.21 (s, 1H, CH, Ar), $7.57(\mathrm{~m}, 2 \mathrm{H},-\mathrm{CH}, \mathrm{Ar}), 7.87(\mathrm{~d}, 2 \mathrm{H}, \mathrm{CH}, \mathrm{Ar})$ and $8.27(\mathrm{~s}$, $1 \mathrm{H}, \mathrm{CH}, \mathrm{Ar}) \mathrm{ppm}$. Elemental analysis $\left(\mathrm{C}_{15} \mathrm{H}_{19} \mathrm{~N}_{3}\right)$; Calcd. C, 74.65; H, 7.94; N, 17.41; found: C, 74.35; H, 7.38; N, 16.36 .

\section{$\mathrm{N}$-(2-(Diethylamino)-ethyl)-quinoline-3-amine(11e)}

Yield: 44\%, m.p: $138-139^{\circ} \mathrm{C}$, FT-IR $\left(\mathrm{KBr}, \mathrm{cm}^{-1}\right): 1130(\mathrm{C}-\mathrm{N}), 1450(\mathrm{C}-\mathrm{H}), 1570(\mathrm{C}=\mathrm{C})$, $1620(\mathrm{C}=\mathrm{N}), 1789(\mathrm{C}-\mathrm{H}), 3293(\mathrm{NH}) .{ }^{1} \mathrm{H}-\mathrm{NMR}$ (DMSO-d $)$ : $\delta 1.45\left(\mathrm{t}, 6 \mathrm{H},\left(-\mathrm{CH}_{3}\right)_{2}\right), 2.67(\mathrm{~m}$, $\left.4 \mathrm{H},\left(-\mathrm{CH}_{2}\right)_{2}\right), 2.83\left(\mathrm{t}, 2 \mathrm{H},-\mathrm{CH}_{2}-\right), 3.37\left(\mathrm{~m}, 2 \mathrm{H},-\mathrm{CH}_{2}-\right), 4.70(\mathrm{t}, 1 \mathrm{H},-\mathrm{NH}), 7.50$ (s, 1H, CH, Ar), $7.63(\mathrm{~m}, 2 \mathrm{H},-\mathrm{CH}, \mathrm{Ar}), 7.85$ (d, 1H, CH, Ar) , 8.13 (d, 1H, CH, Ar) and 8.23 (s, 1H, CH, Ar) 
ppm. Elemental analysis $\left(\mathrm{C}_{15} \mathrm{H}_{21} \mathrm{~N}_{3}\right)$; Calcd. $\mathrm{C}, 74.03 ; \mathrm{H}, 8.70 ; \mathrm{N}, 17.27$; found: $\mathrm{C}, 73.73 ; \mathrm{H}$, $8.09 ; \mathrm{N}, 16.23$.

\section{2-(2-(Quinolin-3-ylamino)-ethyl)-isoindoline-1,3-dione (11f)}

Yield: 38\%, m.p: $129-132{ }^{\circ} \mathrm{C}, \mathrm{FT}-\mathrm{IR}\left(\mathrm{KBr}, \mathrm{cm}^{-1}\right): 1142(\mathrm{C}-\mathrm{N}), 1478(\mathrm{C}-\mathrm{H}), 1514(\mathrm{C}=\mathrm{C})$, $1684(\mathrm{C}=\mathrm{O}), 1927(\mathrm{C}-\mathrm{H}), 3328(\mathrm{~N}-\mathrm{H}) .{ }^{1} \mathrm{H}-\mathrm{NMR}\left(\mathrm{CDCl}_{3}\right): \delta 2.86\left(\mathrm{t}, 2 \mathrm{H},-\mathrm{CH}_{2-}\right), 3.60(\mathrm{~m}, 1 \mathrm{H},-$ $\mathrm{CH}_{2^{-}}$), 5.1 (t, 1H, -NH), 7.57 (s, 1H, CH, Ar), 7.69 (m, 2H, -CH, Ar), 7.89 (d, 1H, CH, Ar), 7.94 (m, 4H, CH, phthalimide), 8.15 (d, 1H, CH, Ar), 8.25 (s, 1H, CH, Ar) and 7.86 (m, 4H, $\mathrm{CH}$, phthalimide) ppm. Elemental analysis $\left(\mathrm{C}_{19} \mathrm{H}_{15} \mathrm{~N}_{3}\right)$; Calcd. C, 71.91; H, 4.76; N, 13.24; found: C, 71.62; H, 4.42; N, 12.44 .

\section{$\mathrm{N}$-(2-(Piperidine-1-yl)-ethyl)-quinoline-6-amine (12a)}

Yield: 37\%, m.p: $158-161^{\circ} \mathrm{C}$, FT-IR $\left(\mathrm{KBr}, \mathrm{cm}^{-1}\right): 1109(\mathrm{C}-\mathrm{N}), 1436(\mathrm{CH}), 1619(\mathrm{C}=\mathrm{C})$, $1745(\mathrm{C}=\mathrm{N}), 3656(\mathrm{~N}-\mathrm{H}) .{ }^{1} \mathrm{H}-\mathrm{NMR}\left(\mathrm{DMSO}-d_{6}\right): \delta 1.71\left(\mathrm{~m}, 6 \mathrm{H},\left(-\mathrm{CH}_{2}-\right)_{2}\right.$, piperidine), $2.46(\mathrm{t}$, $\left.4 \mathrm{H},\left(-\mathrm{NCH}_{2}\right)_{2}\right), 2.66\left(\mathrm{t}, 2 \mathrm{H},-\mathrm{CH}_{2}-\right), 3.32\left(\mathrm{~m}, 2 \mathrm{H},-\mathrm{CH}_{2}-\right), 4.14(\mathrm{t}, 1 \mathrm{H},-\mathrm{NH}), 7.23(\mathrm{~d}, 1 \mathrm{H}, \mathrm{CH}$, Ar), 7.45 (t, 1H, CH, Ar), 7.50 (s, 1H, -CH, Ar), 8.10 (d, 2H, CH, Ar) and 8.73 (d, 1H, CH, Ar) ppm. Elemental analysis $\left(\mathrm{C}_{16} \mathrm{H}_{21} \mathrm{~N}_{3}\right)$; Calcd. C, 75.26; H, 8.29; N, 16.46; found: $\mathrm{C}, 75.00 ; \mathrm{H}$, $7.79 ; \mathrm{N}, 15.63$.

\section{$\mathrm{N}$-(2-(Dimethylamino)-ethyl)-quinoline-6-amine (12b)}

Yield: $40 \%$, m.p: $154-157^{\circ} \mathrm{C}$, FT-IR $\left(\mathrm{KBr}, \mathrm{cm}^{-1}\right): 1214(\mathrm{C}-\mathrm{N}), 1462(\mathrm{CH}), 1575(\mathrm{C}=\mathrm{C})$, $1642(\mathrm{C}=\mathrm{N}), 3309(-\mathrm{NH}-) .{ }^{1} \mathrm{H}-\mathrm{NMR}\left(\mathrm{DMSO}-d_{6}\right): \delta 2.28\left(\mathrm{~s}, 6 \mathrm{H},\left(-\mathrm{CH}_{3}\right)_{2}\right), 2.71\left(\mathrm{t}, 2 \mathrm{H},-\mathrm{CH}_{2}-\right)$, $3.29\left(\mathrm{~m}, 2 \mathrm{H},-\mathrm{CH}_{2^{-}}\right), 4.51(\mathrm{t}, 1 \mathrm{H},-\mathrm{NH}), 7.23(\mathrm{~d}, 1 \mathrm{H}, \mathrm{CH}, \mathrm{Ar}), 7.39$ (t, 1H, CH, Ar), 7.57 (s, 1H, $-\mathrm{CH}, \mathrm{Ar}), 8.14(\mathrm{~d}, 2 \mathrm{H}, \mathrm{CH}, \mathrm{Ar})$ and $8.69(\mathrm{~d}, 1 \mathrm{H}, \mathrm{CH}, \mathrm{Ar}) \mathrm{ppm}$. Elemental analysis $\left(\mathrm{C}_{13} \mathrm{H}_{17} \mathrm{~N}_{3}\right)$; Calcd. C, 72.52; H, 7.96; N, 19.52; found: C, 72.30; H, 7.48; N, 18.54 .

\section{$\mathrm{N}$-(2-Morpholinoethyl)-quinoline-6-amine (12c)}

Yield: 39\%, m.p: $163-166^{\circ} \mathrm{C}, \mathrm{FT}-\mathrm{IR}\left(\mathrm{KBr}, \mathrm{cm}^{-1}\right): 1022(\mathrm{C}-\mathrm{O}), 1100(\mathrm{C}-\mathrm{N}), 1471(\mathrm{C}=\mathrm{C})$, $1594(\mathrm{C}=\mathrm{N}), 3697(-\mathrm{NH}-) .{ }^{1} \mathrm{H}-\mathrm{NMR}\left(\mathrm{CDCl}_{3}\right): \delta 2.47\left(\mathrm{t}, 4 \mathrm{H},\left(-\mathrm{NCH}_{2}\right)_{2}\right.$ morpholine), $2.66(\mathrm{t}$, $\left.2 \mathrm{H},-\mathrm{CH}_{2}-\right), 3.37\left(\mathrm{~m}, 2 \mathrm{H},-\mathrm{CH}_{2}-\right), 3.59$ (t, $4 \mathrm{H},\left(-\mathrm{OCH}_{2}\right)_{2}$ morpholine), 4.80 (t, 1H, -NH), 7.24 (d, 1H, CH, Ar), 7.43 (t, 1H, CH, Ar), 7.48 (s, 1H, -CH, Ar), 8.11 (d, 2H, CH, Ar) and 8.68 (d, $1 \mathrm{H}, \mathrm{CH}, \mathrm{Ar}) \mathrm{ppm}$. Elemental analysis $\left(\mathrm{C}_{15} \mathrm{H}_{19} \mathrm{~N}_{3}\right)$; Calcd. C, 70.01; H, 7.44; N, 16.33; found: C, 69.79; H, 6.99; N, 15.51 .

\section{$\mathrm{N}$-(2-(Pyrrolidine-1-yl)-ethyl)-quinoline-6-amine (12d)}

Yield: 44\%, m.p: $144-14{ }^{\circ} \mathrm{C}$, FT-IR (KBr, cm$\left.{ }^{-1}\right): 1058$ (C-N), 1257 (C-N), 1530 (C$\mathrm{H}), 1637(\mathrm{C}=\mathrm{C}), 1664(\mathrm{C}=\mathrm{N}), 1775(\mathrm{C}-\mathrm{H}) .{ }^{1} \mathrm{H}-\mathrm{NMR}\left(\mathrm{DMSO}-d_{6}\right): \delta 1.14\left(\mathrm{~m}, 4 \mathrm{H},\left(-\mathrm{CH}_{2}-\right)_{2}\right.$ pyrrolidine), $1.23\left(\mathrm{t}, 2 \mathrm{H},-\mathrm{CH}_{2}-\right), 1.89$ (t, $4 \mathrm{H},\left(-\mathrm{NCH}_{2}\right)_{2}$, pyrrolidine), $2.39\left(\mathrm{~m}, 2 \mathrm{H},-\mathrm{CH}_{2}-\right), 3.50$ (t, 1H, -NH), 7.22 (d, 1H, CH, Ar), 7.42 (t, 1H, CH, Ar), 7.59 (s, 1H, -CH, Ar), 8.12 (d, 2H, $\mathrm{CH}, \mathrm{Ar})$ and $8.71(\mathrm{~d}, 1 \mathrm{H}, \mathrm{CH}, \mathrm{Ar}) \mathrm{ppm}$. Elemental analysis $\left(\mathrm{C}_{15} \mathrm{H}_{19} \mathrm{~N}_{3}\right)$; Calcd. C, 74.65; H, 7.94; N, 17.41; found: C, 74.42; H, 7.46; N, 16.53 .

\section{$\mathrm{N}$-(2-(Diethylamino)-ethyl)-quinoline-6-amine (12e)}

Yield: 47\%, m.p: $168-171^{\circ} \mathrm{C}$, FT-IR (KBr, cm $\left.{ }^{-1}\right): 1050(\mathrm{C}-\mathrm{N}), 1150(\mathrm{C}-\mathrm{N}), 1380$ (C$\mathrm{N}), 1480(\mathrm{C}-\mathrm{H}), 1575(\mathrm{C}=\mathrm{N}), 1660(\mathrm{C}=\mathrm{C}), 3280(\mathrm{~N}-\mathrm{H}) .{ }^{1} \mathrm{H}-\mathrm{NMR}\left(\mathrm{DMSO}-d_{6}\right): \delta 1.18(\mathrm{t}, 6 \mathrm{H},(-$ $\left.\left.\mathrm{CH}_{3}\right)_{2}\right), 2.36\left(\mathrm{~m}, 4 \mathrm{H},\left(\mathrm{NCH}_{2}\right)_{2}\right), 2.72\left(\mathrm{t}, 2 \mathrm{H},-\mathrm{CH}_{2}-\right), 3.31\left(\mathrm{~m}, 2 \mathrm{H},-\mathrm{CH}_{2}-\right), 3.0(\mathrm{t}, 1 \mathrm{H},-\mathrm{NH}), 7.25$ (d, 1H, CH, Ar), 7.37 (t, 1H, CH, Ar), 7.52 (s, 1H, -CH, Ar), 8.13 (d, 2H, CH, Ar) and 8.73 (d, $1 \mathrm{H}, \mathrm{CH}, \mathrm{Ar}) \mathrm{ppm}$. Elemental analysis $\left(\mathrm{C}_{15} \mathrm{H}_{21} \mathrm{~N}_{3}\right)$; Calcd. C, 74.03; H, 8.70; N, 17.27; found: C, 73.80; H, 8.17; N, 16.40 .

\section{2-(2-(Quinolin-6-ylamino)-ethyl)-isoindoline-1,3-dione (12f)}

Yield: 43\%, m.p: 162-165 ${ }^{\circ} \mathrm{C}$, FT-IR $\left(\mathrm{KBr}, \mathrm{cm}^{-1}\right): 1157(\mathrm{C}-\mathrm{N}), 1468(\mathrm{C}-\mathrm{H}), 1606(\mathrm{C}=\mathrm{C})$, $1673(\mathrm{C}=\mathrm{N}), 1738(\mathrm{C}=\mathrm{O}) .{ }^{1} \mathrm{H}-\mathrm{NMR}\left(\mathrm{CDCl}_{3}\right): \delta 3.88\left(\mathrm{~m}, 2 \mathrm{H},-\mathrm{CH}_{2^{-}}\right), 3.38\left(\mathrm{t}, 2 \mathrm{H},-\mathrm{CH}_{2^{-}}\right), 4.90$ (t, 1H, -NH), 7.25 (d, 1H, CH, Ar), 7.44 (t, 1H, CH, Ar), 7.56 (s, 1H, -CH, Ar), 8.14 (d, 2H, 
$\mathrm{CH}, \mathrm{Ar}), 8.76(\mathrm{~d}, 1 \mathrm{H}, \mathrm{CH}, \mathrm{Ar})$ and $7.86(\mathrm{~m}, 4 \mathrm{H}, \mathrm{CH}$, phthalimide) ppm. Elemental analysis $\left(\mathrm{C}_{19} \mathrm{H}_{15} \mathrm{~N}_{3}\right)$; Calcd. C, 71.91; H, 4.76; N, 13.24; found: C, 71.69; H, 4.47; N, 12.57 .

\section{$\mathrm{N}$-(2-(Piperidine-1-yl)-ethyl)-quinoline-8-amine (13a)}

Yield: 39\%, m.p: $108-111^{\circ} \mathrm{C}$, FT-IR $\left(\mathrm{KBr}, \mathrm{cm}^{-1}\right): 1140(\mathrm{C}-\mathrm{N}), 1487(-\mathrm{CH}), 1520(\mathrm{C}=\mathrm{C})$, $1634(\mathrm{C}=\mathrm{N}), 3601(-\mathrm{NH}-) .{ }^{1} \mathrm{H}-\mathrm{NMR}\left(\mathrm{DMSO}-d_{6}\right): \delta 1.69\left(\mathrm{~m}, 6 \mathrm{H},\left(-\mathrm{CH}_{2}-\right)_{2}\right), 2.46(\mathrm{t}, 4 \mathrm{H},(-$ $\left.\left.\mathrm{NCH}_{2}\right)_{2}\right), 2.67\left(\mathrm{t}, 2 \mathrm{H},-\mathrm{CH}_{2}-\right), 3.39\left(\mathrm{~m}, 2 \mathrm{H},-\mathrm{CH}_{2}-\right), 4.33(\mathrm{t}, 1 \mathrm{H},-\mathrm{NH}), 7.29$ (d, 1H, CH, Ar), $7.50(\mathrm{~m}, 2 \mathrm{H}, \mathrm{CH}, \mathrm{Ar})$ and $8.27(\mathrm{~m}, 3 \mathrm{H}, \mathrm{CH}, \mathrm{Ar}) \mathrm{ppm}$. Elemental analysis $\left(\mathrm{C}_{16} \mathrm{H}_{21} \mathrm{~N}_{3}\right)$; Calcd. C, 75.26; H, 8.29; N, 16.46; found: C, 75.10; H, 7.87; N, 15.80 .

\section{$\mathrm{N}$-(2-(Dimethylamino)-ethyl)-quinoline-8-amine (13b)}

Yield: 37\%, m.p: $119-122^{\circ} \mathrm{C}$, FT-IR $\left(\mathrm{KBr}, \mathrm{cm}^{-1}\right): 1214(\mathrm{C}-\mathrm{N}), 1462(\mathrm{CH}), 1522(\mathrm{C}=\mathrm{C})$, $1730(\mathrm{C}=\mathrm{N}), 3369(-\mathrm{NH}-) .{ }^{1} \mathrm{H}-\mathrm{NMR}\left(\mathrm{DMSO}-d_{6}\right): \delta 2.27\left(\mathrm{~s}, 6 \mathrm{H},\left(-\mathrm{CH}_{3}\right)_{2}\right), 2.72\left(\mathrm{t}, 2 \mathrm{H},-\mathrm{CH}_{2}-\right)$, $3.35\left(\mathrm{t}, 2 \mathrm{H},-\mathrm{CH}_{2}-\right), 4.37(\mathrm{t}, 1 \mathrm{H},-\mathrm{NH}), 7.52(\mathrm{~m}, 4 \mathrm{H}, \mathrm{CH}, \mathrm{Ar})$ and $8.23(\mathrm{~d}, 2 \mathrm{H}, \mathrm{CH}, \mathrm{Ar}) \mathrm{ppm}$. Elemental analysis $\left(\mathrm{C}_{13} \mathrm{H}_{17} \mathrm{~N}_{3}\right)$; Calcd. $\mathrm{C}, 72.52 ; \mathrm{H}, 7.96 ; \mathrm{N}, 19.52$; found: $\mathrm{C}, 72.37 ; \mathrm{H}, 7.56$; N, 18.73.

\section{$\mathrm{N}$-(2-Morpholinoethyl)-quinoline-8-amine (13c)}

Yield: 41\%, m.p: $113-116^{\circ} \mathrm{C}, \mathrm{FT}-\mathrm{IR}\left(\mathrm{KBr}, \mathrm{cm}^{-1}\right): 1083$ (C-O), $1122(\mathrm{C}-\mathrm{N}), 1451$ (CH2), $1570(\mathrm{C}=\mathrm{C}), 1676(\mathrm{C}=\mathrm{N}) .{ }^{1} \mathrm{H}-\mathrm{NMR}\left(\mathrm{CDCl}_{3}\right): \delta 2.48\left(\mathrm{t}, 4 \mathrm{H},\left(-\mathrm{NCH}_{2}\right)_{2}\right.$, morpholine $), 2.69(\mathrm{t}$, $\left.2 \mathrm{H},-\mathrm{CH}_{2}-\right), 3.38\left(\mathrm{~m}, 2 \mathrm{H},-\mathrm{CH}_{2}-\right), 3.62\left(\mathrm{t}, 4 \mathrm{H},\left(-\mathrm{OCH}_{2}\right)_{2}\right.$, morpholine $), 4.80(\mathrm{t}, 1 \mathrm{H},-\mathrm{NH}), 7.28$ (d, 1H, CH, Ar), 7.44 (t, 1H, -CH, Ar), 7.47 (t, 1H, CH, Ar), $7.85(\mathrm{~d}, 2 \mathrm{H}, \mathrm{CH}, \mathrm{Ar})$ and $8.74(\mathrm{~d}$, $1 \mathrm{H}, \mathrm{CH}, \mathrm{Ar}) \mathrm{ppm}$. Elemental analysis $\left(\mathrm{C}_{15} \mathrm{H}_{19} \mathrm{~N}_{3}\right)$; Calcd. C, 70.01; H, 7.44; N, 16.33; found: C, 69.87; H, 7.06; N, 15.67.

\section{$\mathrm{N}$-(2-(Pyrrolidine-1-yl)-ethyl)-quinoline-8-amine (13d)}

Yield: $43 \%$, m.p: 106-109 ${ }^{\circ} \mathrm{C}$, FT-IR $\left(\mathrm{KBr}, \mathrm{cm}^{-1}\right): 1463(\mathrm{C}-\mathrm{H}), 1570(\mathrm{C}=\mathrm{C})$, 1665(C=N), $1960(\mathrm{C}-\mathrm{H}) .{ }^{1} \mathrm{H}-\mathrm{NMR}$ (DMSO- $\left.d_{6}\right): \delta 1.83\left(\mathrm{~m}, 4 \mathrm{H},\left(-\mathrm{CH}_{2}-\right)_{2}\right.$, pyrrolidine), 2.67 (t, $\left.2 \mathrm{H},-\mathrm{CH}_{2}-\right), 2.82\left(\mathrm{t}, 4 \mathrm{H},\left(-\mathrm{NCH}_{2}\right)_{2}\right.$, pyrrolidine), $3.41\left(\mathrm{~m}, 2 \mathrm{H},-\mathrm{CH}_{2}-\right), 3.25(\mathrm{t}, 1 \mathrm{H},-\mathrm{NH}), 7.43$ $(\mathrm{m}, 5 \mathrm{H},-\mathrm{CH}, \mathrm{Ar})$ and $8.19(\mathrm{~d}, 1 \mathrm{H}, \mathrm{CH}, \mathrm{Ar}) \mathrm{ppm}$. Elemental analysis $\left(\mathrm{C}_{15} \mathrm{H}_{19} \mathrm{~N}_{3}\right)$; Calcd. $\mathrm{C}$, 74.65; H, 7.94; N, 17.41; found: C, 74.5; H, 7.54; N, 16.71 .

\section{$\mathrm{N}$-(2-(Diethylamino)-ethyl)-quinoline-8-amine (13e)}

Yield: 48\%, m.p: $123-126^{\circ} \mathrm{C}$, FT-IR (KBr, cm$\left.{ }^{-1}\right): 1212(\mathrm{C}-\mathrm{N}), 1478(\mathrm{C}-\mathrm{H}), 1605(\mathrm{C}=\mathrm{C})$, $1670(\mathrm{C}=\mathrm{N}), 1768(\mathrm{C}-\mathrm{H}) .{ }^{1} \mathrm{H}-\mathrm{NMR}\left(\mathrm{DMSO}-d_{6}\right): \delta 1.12\left(\mathrm{t}, 6 \mathrm{H},\left(-\mathrm{CH}_{3}\right)_{2}\right), 3.45(\mathrm{t}, 1 \mathrm{H},-\mathrm{NH})$, $2.57\left(\mathrm{~m}, 4 \mathrm{H},\left(-\mathrm{NCH}_{2}\right)_{2}\right), 2.69\left(\mathrm{t}, 2 \mathrm{H},-\mathrm{CH}_{2}-\right), 3.32\left(\mathrm{~m}, 2 \mathrm{H},-\mathrm{CH}_{2}-\right), 7.40(\mathrm{~m}, 5 \mathrm{H},-\mathrm{CH}, \mathrm{Ar})$ and $8.72(\mathrm{~d}, 1 \mathrm{H}, \mathrm{CH}, \mathrm{Ar}) \mathrm{ppm}$. Elemental analysis $\left(\mathrm{C}_{15} \mathrm{H}_{21} \mathrm{~N}_{3}\right)$; Calcd. C, 74.03; H, 8.70; N, 17.27; found: C, 73.88; H, 8.26; N, 16.57 .

\section{2-(2-(Quinolin-8-ylamino)-ethyl)-isoindoline-1,3-dione (13f)}

Yield: 54\%, m.p: $116-119{ }^{\circ} \mathrm{C}$, FT-IR (KBr, cm $\left.{ }^{-1}\right): 1172$ (C-N), 1442 (C-H), 1580 $(\mathrm{C}=\mathrm{C}), 1658(\mathrm{C}=\mathrm{N}) .{ }^{1} \mathrm{H}-\mathrm{NMR}\left(\mathrm{CDCl}_{3}\right): \delta 3.57\left(\mathrm{~m}, 2 \mathrm{H},-\mathrm{CH}_{2}\right), 3.90\left(\mathrm{t}, 2 \mathrm{H},-\mathrm{CH}_{2}-\right), 4.41(\mathrm{t}, 1 \mathrm{H}$, -NH), 7.25 (d, 1H, CH, Ar), 7.46 (m, 2H, -CH, Ar), 7.69 (d, 1H, CH, Ar), 7.88 (m, 4H, CH, , phthalimide) and $8.57(\mathrm{~d}, 2 \mathrm{H}, \mathrm{CH}, \mathrm{Ar}) \mathrm{ppm}$. Elemental analysis $\left(\mathrm{C}_{19} \mathrm{H}_{15} \mathrm{~N}_{3}\right)$; Calcd. C, 71.91; $\mathrm{H}, 4.76$; N, 13.24; found: C, 71.76; H, 4.52; N, 12.71 .

\subsection{Biological activity.}

Resistance of different strains of Leishmania species to conventional antileishmanial treatment has emerged as one of the world's most pressing issues. Thus, the development of new heterocyclic compounds having substantial biological and medicinal importance is a key objective of our study. Accordingly, various synthesized derivatives were screened for their invitro antileishmanial activity against the promastigote form of Leishmania donovani. The 
inhibitory concentration that reduces the parasitic growth by 50\% (IC50), as listed in Table 1, was calculated by projecting percentage inhibition against the concentration of drug-using statistical analysis and the parasite inhibition curve of compounds (11a-f, 12a-f, and 13a-13f), and sitamaquine is shown in Figure 3.

Table 1. $\mathrm{IC}_{50}$ of newly synthesized quinoline-based compounds.

\begin{tabular}{l|c|c}
\multicolumn{1}{c|}{ S. No. } & Compound No. & IC $_{\mathbf{5 0}}(\mu \mathrm{g} / \mathrm{mL})$ \\
\hline 1. & $11 \mathrm{a}$ & 17.73 \\
\hline 2. & $11 \mathrm{~b}$ & 30.94 \\
\hline 3. & $11 \mathrm{c}$ & 18.83 \\
\hline 4. & $11 \mathrm{~d}$ & 21.06 \\
\hline 5. & $11 \mathrm{e}$ & 39.71 \\
\hline 6. & $11 \mathrm{f}$ & 13.61 \\
\hline 7. & $12 \mathrm{a}$ & 23.08 \\
\hline 8. & $12 \mathrm{~b}$ & 35.79 \\
\hline 9. & $12 \mathrm{c}$ & 21.70 \\
\hline 10. & $12 \mathrm{~d}$ & 23.52 \\
\hline 11. & $12 \mathrm{e}$ & 40.76 \\
\hline 12. & $12 \mathrm{f}$ & 11.92 \\
\hline 13. & $13 \mathrm{a}$ & 17.47 \\
\hline 14. & $13 \mathrm{~b}$ & 26.44 \\
\hline 15. & $13 \mathrm{c}$ & 17.87 \\
\hline 16. & $13 \mathrm{~d}$ & 23.23 \\
\hline 17. & $13 \mathrm{e}$ & 30.55 \\
\hline 18. & $13 \mathrm{f}$ & 10.41 \\
\hline 19. & Sitamaquine & 10.09
\end{tabular}

The results of antileishmanial activity elucidated that most of the derivatives demonstrated good activity against promastigote form of Leishmania donovani with $\mathrm{IC}_{50}$ value ranging from 10.41 to $40.76 \mu \mathrm{g} / \mathrm{ml}$ as compared to standard sitamaquine with $\mathrm{IC}_{50}$ value of $10.09 \mu \mathrm{g} / \mathrm{ml}$.
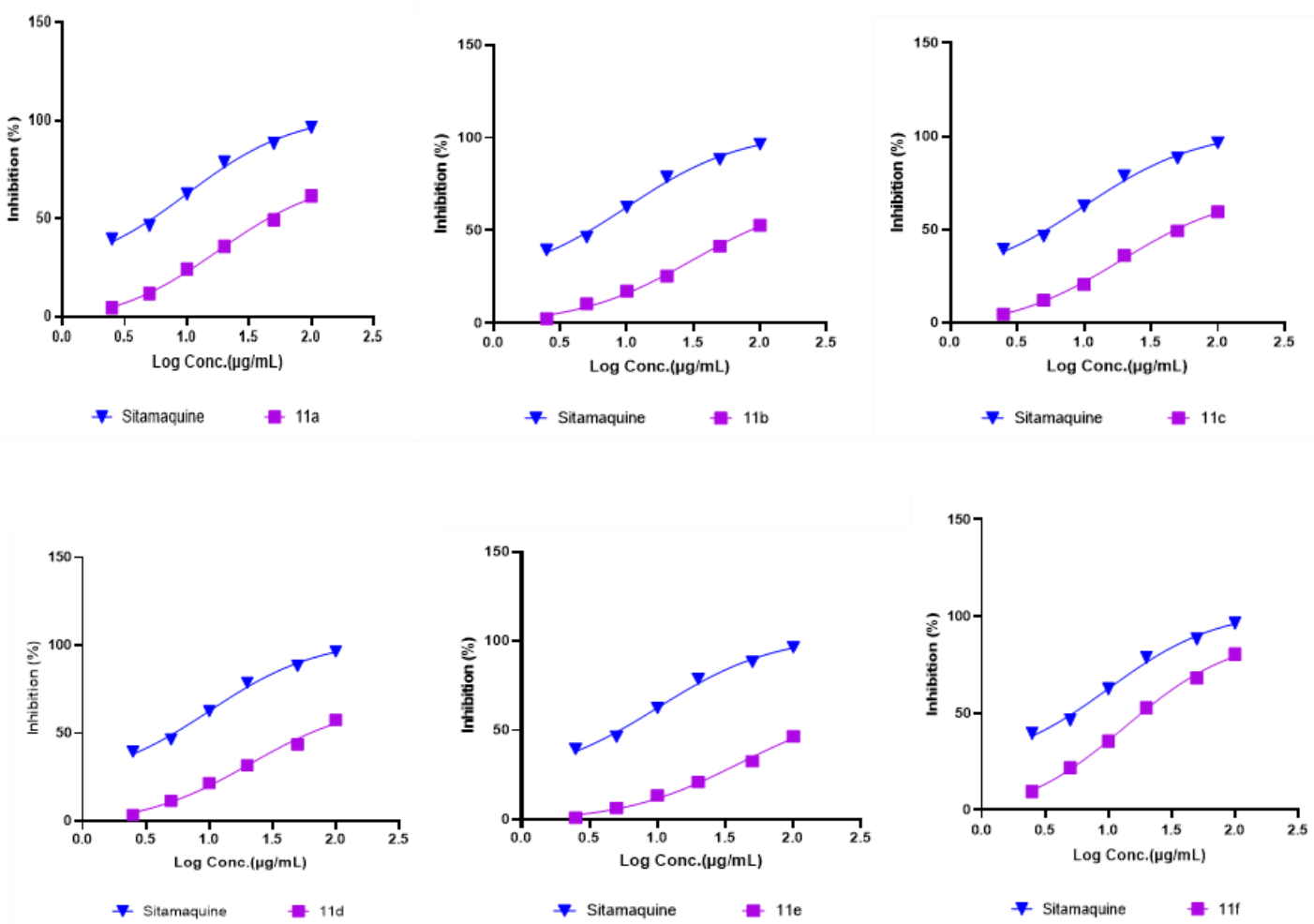

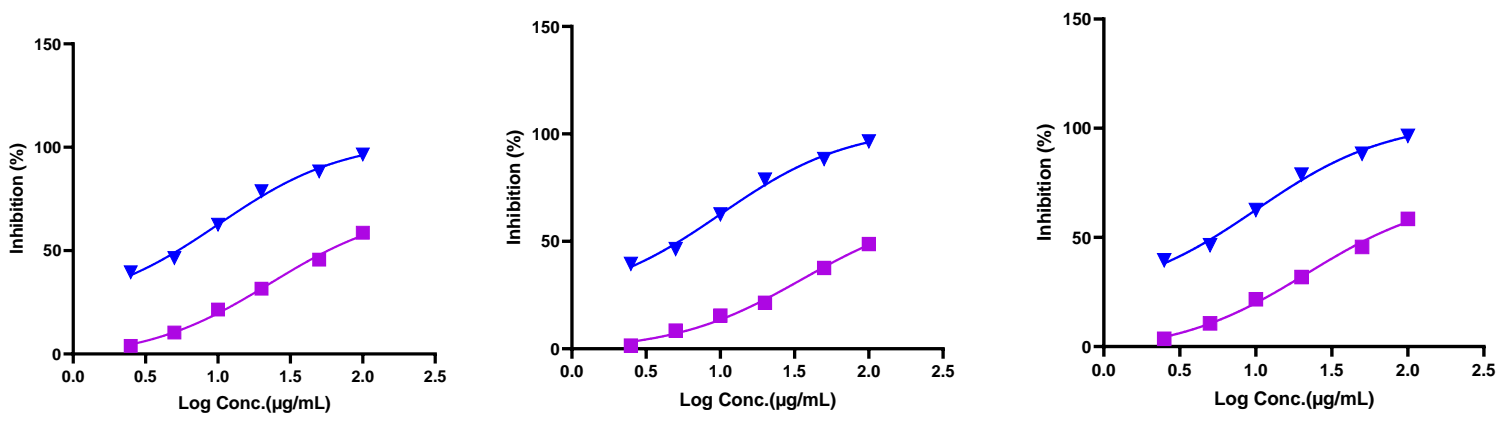

$\rightarrow$ Sitamaquine $\quad \rightarrow 12 \mathrm{a}$

$\mp$ Sitamaquine $\quad+12 \mathrm{~b}$
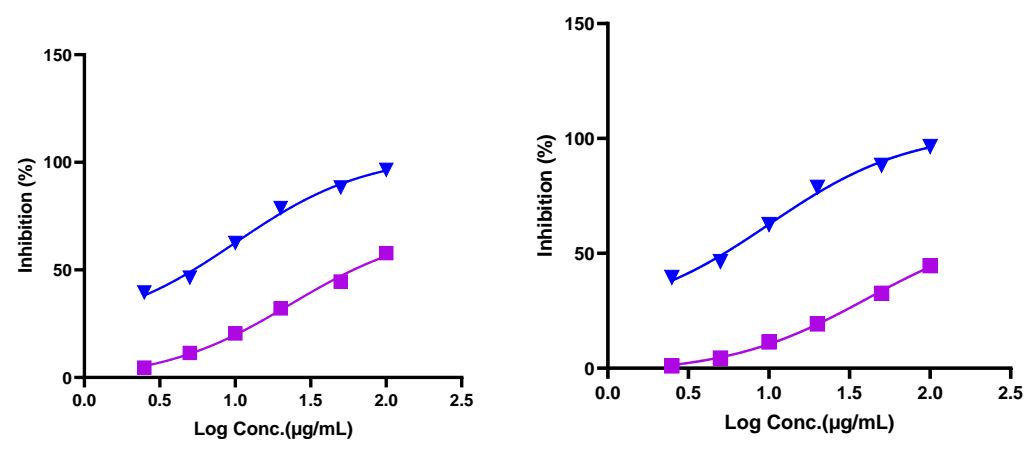

$\mp$ Sitamaquine $\quad-\quad 12 \mathrm{c}$

$\rightarrow$ Sitamaquine $\quad+12 \mathrm{~d}$

$\mp$ Sitamaquine $\quad+712 e$

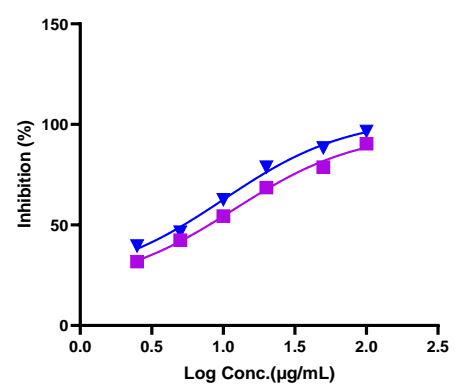

- Sitamaquine $\quad+$ - 12
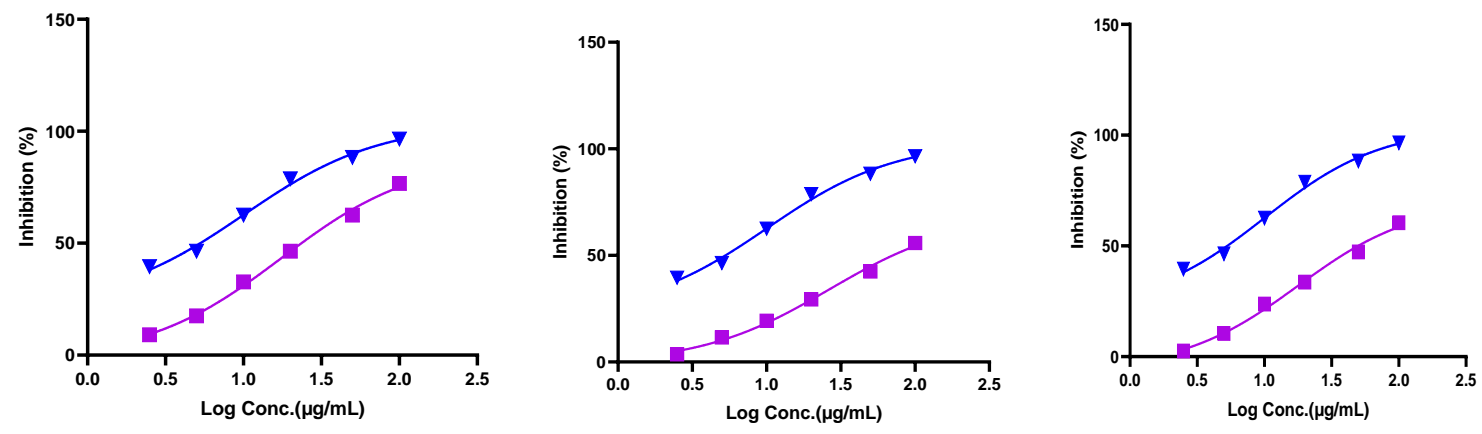

$\rightarrow$ Sitamaquine $\quad+73$

₹ Sitamaquine

$+13 \mathrm{~b}$

† Sitamaquine $\quad+$ 13c
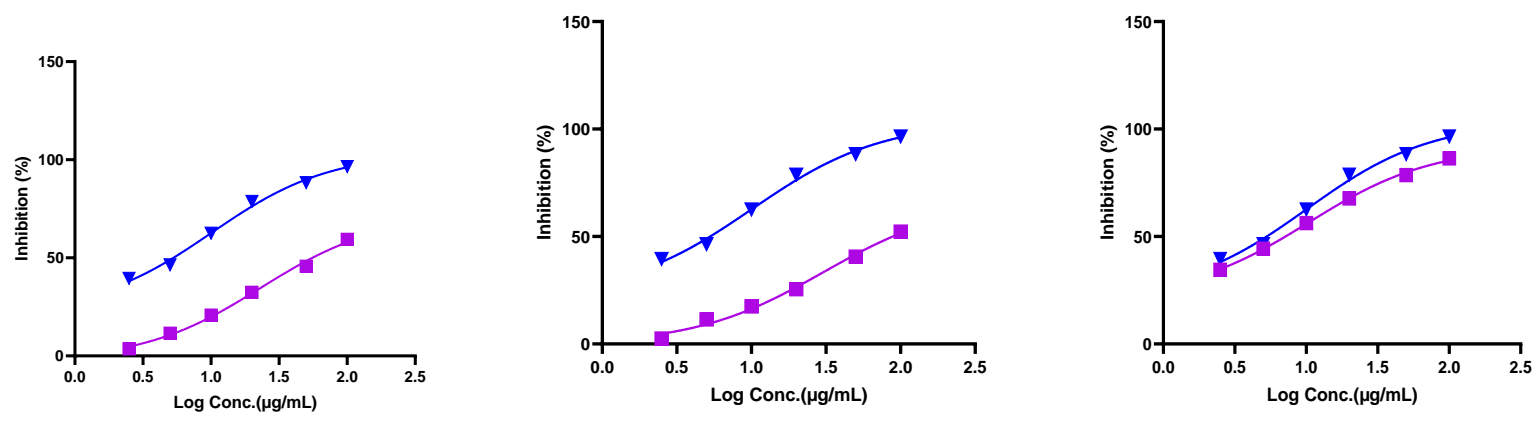

\# Sitamaquine $\quad-$ - $13 d$

F Sitamaquine

늘 $13 \mathrm{e}$

$\rightarrow$ Sitamaquine $\quad-13$

Figure 3. The parasite inhibition curve of compounds 11a-f, 12a-f, and 13a-f.

Amid the synthetic derivatives, the compounds 11f, 12f and $13 f$ displayed comparable activity to standard with $\mathrm{IC}_{50}$ values of $13.61,11.92,10.41$, whereas compounds $11 \mathrm{a}, 11 \mathrm{c}, 13 \mathrm{a}$, and $13 \mathrm{c}$ exhibit moderate activity with $\mathrm{IC}_{50}$ values of $17.73,18.83,17.47$, and 17.87 . On the other hand, the rest of the compounds exhibit inferior activity as compared to the standard. 


\subsection{Molecular docking.}

The molecular docking procedure was used to predict the binding interactions between the quinoline derivatives and the binding pocket of the enzyme adenine phosphoribosyltransferase from Leishmania donovani for further pharmacological evaluation. Binding energy $\left(\mathrm{kcal} / \mathrm{mol}^{-1}\right)$ is the significant key parameter of molecular docking that indicates the affinity and intensity of the interaction between the ligand and the receptor. The binding energy and intensity of interaction are reciprocal to each other, i.e., and the more is the binding energy, the less will be the intensity of the interaction and vice versa. Therefore, during docking studies, the ligand that displays the minimal binding energy is considered ideally suited for further evaluation.

All the docked conformations were analyzed, and the best-scored pose for each compound was selected for further interaction studies. The docking scores of various compounds are given in Table 2.

Table 2. Docking studies of the quinoline-based compounds.

\begin{tabular}{|c|c|c|c|}
\hline S.No. & $\begin{array}{l}\text { Compound } \\
\text { Name }\end{array}$ & 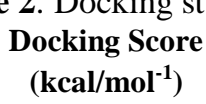 & Probable interacting Residues \\
\hline 1. & $11 \mathrm{a}$ & -6.6 & Ser157, Leu156, Met129, Glu127, Pro126, Pro1 16 \\
\hline 2. & $11 \mathrm{~b}$ & -6.0 & Leu156, Glu127, Pro126 \\
\hline 3. & $11 \mathrm{c}$ & -6.6 & Ser157, Leu156, Pro126 \\
\hline 4. & $11 \mathrm{~d}$ & -6.5 & Ser157, Leu156, Met129, Glu127, Pro126, Phe79 \\
\hline 5. & $11 \mathrm{e}$ & -5.8 & $\begin{array}{l}\text { Ser157, Leu156, Ala155, Leu149, Glu127, Pro126, Pro116, } \\
\text { Phe79 }\end{array}$ \\
\hline 6. & $11 \mathrm{f}$ & -7.2 & Ile187, Ala183, Ser157, Ala155, Leu156, Pro126 \\
\hline 7. & $12 \mathrm{a}$ & -6.4 & Lys186, Leu156, Pro126, Glu120, Glu118, Pro116 \\
\hline 8. & $12 \mathrm{~b}$ & -5.9 & Ser157, Leu156, Glu127, Pro126 \\
\hline 9. & $12 \mathrm{c}$ & -6.5 & Ser157, Leu156, Glu127, Pro126, Asp80 \\
\hline 10. & $12 \mathrm{~d}$ & -6.4 & Ser157, Leu156, Met129, Glu127, Pro126, Phe79 \\
\hline 11. & $12 \mathrm{e}$ & -5.8 & Ile187, Lys186, Ala183, Ser157, Leu156, Pro126 \\
\hline 12. & $12 \mathrm{f}$ & -7.6 & $\begin{array}{l}\text { Ser157, Leu156, Ala155, Asp146, Glu127, Pro126, Asp80, } \\
\text { Phe79 }\end{array}$ \\
\hline 13. & $13 \mathrm{a}$ & -6.6 & Ile187, Ala183, Leu156, Ala155, Pro126, Pro116 \\
\hline 14. & $13 \mathrm{~b}$ & -6.2 & Ser157, Leu156, Glu127, Pro126, Pro116 \\
\hline 15. & $13 \mathrm{c}$ & -6.6 & Ser157, Leu156, Pro126, Pro116, Asp80 \\
\hline 16. & $13 \mathrm{~d}$ & -6.4 & Ser157, Leu156, Met129, Glu127, Pro126, Pro116, Phe79 \\
\hline 17. & $13 \mathrm{e}$ & -6.0 & Ala183, Leu156, Pro126 \\
\hline 18. & $13 \mathrm{f}$ & -7.4 & Lys186, Ala183, Leu156, Pro126, Glu120, Tyr117 \\
\hline 19. & Sitamaquine & -6.9 & Ser157, Leu156, Pro126, Tyr117, Pro116 \\
\hline
\end{tabular}

The outcome of the docking results showed that all the compounds were well acclimatized in the active site of the Leishmania enzyme. Among the various quinolone derivatives, the compound $\mathbf{1 2 f}$ was found to be the most active with a docking score of -7.6 followed by other compounds such as $\mathbf{1 3 f}$ and 11f with docking scores of -7.4 and -7.2, respectively. From the docking conformations of most potent compounds among series, the docking pose of 12f shows three hydrogen bond interactions with Asp80, Ala155, and Leu156 amino acid residues of the target. Among these, Asp80 exhibits conventional hydrogen bonding with the nitrogen of the quinoline ring, whereas the other two form hydrogen bonding with the doubly bonded oxygen atom of the phthalimide ring. The carbon atom of the alkyl chain connecting the quinoline ring with phthalimide moiety exhibit carbon-hydrogen bonding with Glu127 and Ser157. The cyclic ring of quinoline moiety shows Pi anion with Phe79 and pi-pi stacked interactions with Glu127 and Asp146, respectively. The five-membered rings of phthalimide display pi-alkyl interactions with Leu156, whereas six-membered rings of 
phthalimide moiety exhibit pi-sigma and pi-alkyl interactions with Leu156 and Pro126, respectively.

The docking pose of the second-highest compound, i.e., 13f from the series, displays two hydrogen bonding with Tyr117 and Glu120. The nitrogen-containing ring of quinoline moiety exhibited alkyl linkage with Leu156 and Lys186, whereas the other ring of quinoline exhibits pi-alkyl and alkyl linkage with Leu156 and Lys186, respectively. The phthalimide moiety similarly displays alkyl linkage with Pro126 and Leu156.

The docking pose of $\mathbf{1 1 f}$ manifests three hydrogen bond interactions with Ala155, Leu156, and Ser157 amino acid residues of the target. Ser157 among these forms' conventional hydrogen bonding with the nitrogen of quinoline moiety whereas other two form hydrogen bonding with the doubly bonded oxygen atom of phthalimide ring. The cyclic ring of quinoline moiety forms a pi-alkyl linkage with Pro126 and Leu156. The six-membered rings of phthalimide form pi-alkyl linkage with Ala183, Lys186, and Ile187, whereas five-membered heterocyclic rings reveal alkyl linkage with Alkyl Ala155 and Ile187.

The docking conformation of standard drug sitamaquine exhibits conventional hydrogen bonding as well as alkyl/pi-alkyl interactions with the various active residues of the target molecule. The nitrogen of substituted secondary amine moiety of sitamaquine exhibit hydrogen bonding with the Tyr117, where other residue Ser157 showed hydrogen bonding with the substituted oxygen moiety available at sixth carbon of quinoline ring. Other residues such as Pro116, Pro126, and Leu156 displayed alkyl interactions with the sitamaquine. Among these, Leu156 exhibits alkyl interactions with the quinoline ring and alkyl chain of the ligand, whereas Pro116 and Pro126 exhibit alkyl interactions with the methyl and alkyl chain of the ligand.

Among the remaining derivatives, the compounds 11a, 11c, 13a, 13c, and 13d, showed comparable binding affinity compared to standard drug sitamaquine. The docking pose and ligand interactions along with bonding types of various derivatives are given in Table 3.

Table 3. Binding surface and ligand-receptor interaction of various compounds.

\begin{tabular}{|c|c|c|c|c|}
\hline S.No & Compd No. & Binding Surface & Ligand Interactions & Bonding \\
\hline 1. & $11 \mathrm{a}$ & & & $\begin{array}{l}\text { Conventional hydrogen } \\
\text { bonding, Vander wall, } \\
\text { and pi-alkyl interactions }\end{array}$ \\
\hline 2. & $11 b$ & & $\approx$ & $\begin{array}{l}\text { Vander wall, pi-sigma, } \\
\text { and pi-alkyl interactions }\end{array}$ \\
\hline 3. & $11 \mathrm{c}$ & & & $\begin{array}{l}\text { pi-sigma, pi-alkyl, and } \\
\text { unfavorable donor-donor } \\
\text { interactions }\end{array}$ \\
\hline
\end{tabular}


https://doi.org/10.33263/BRIAC126.75037522

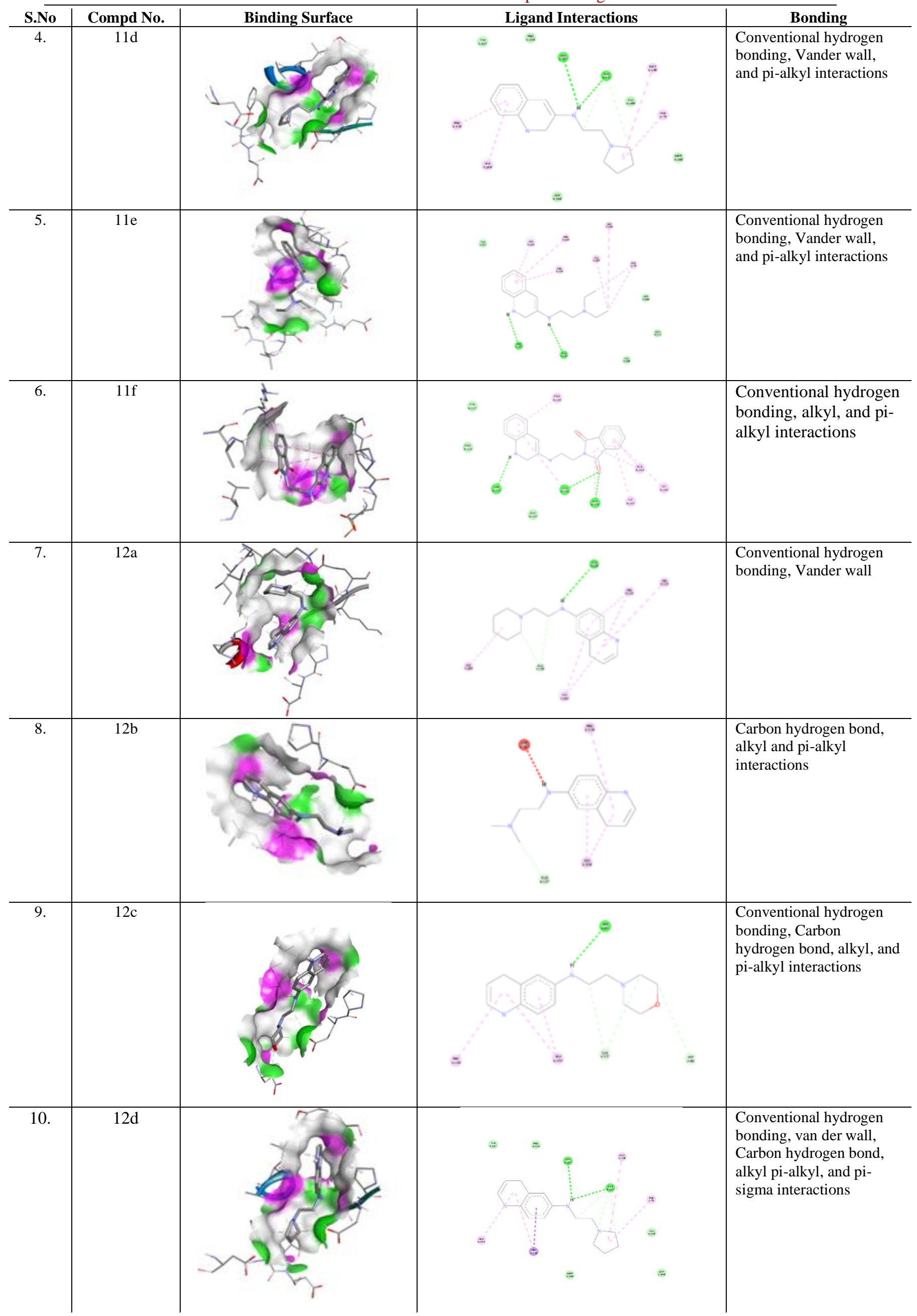

https://biointerfaceresearch.com/ 
https://doi.org/10.33263/BRIAC126.75037522

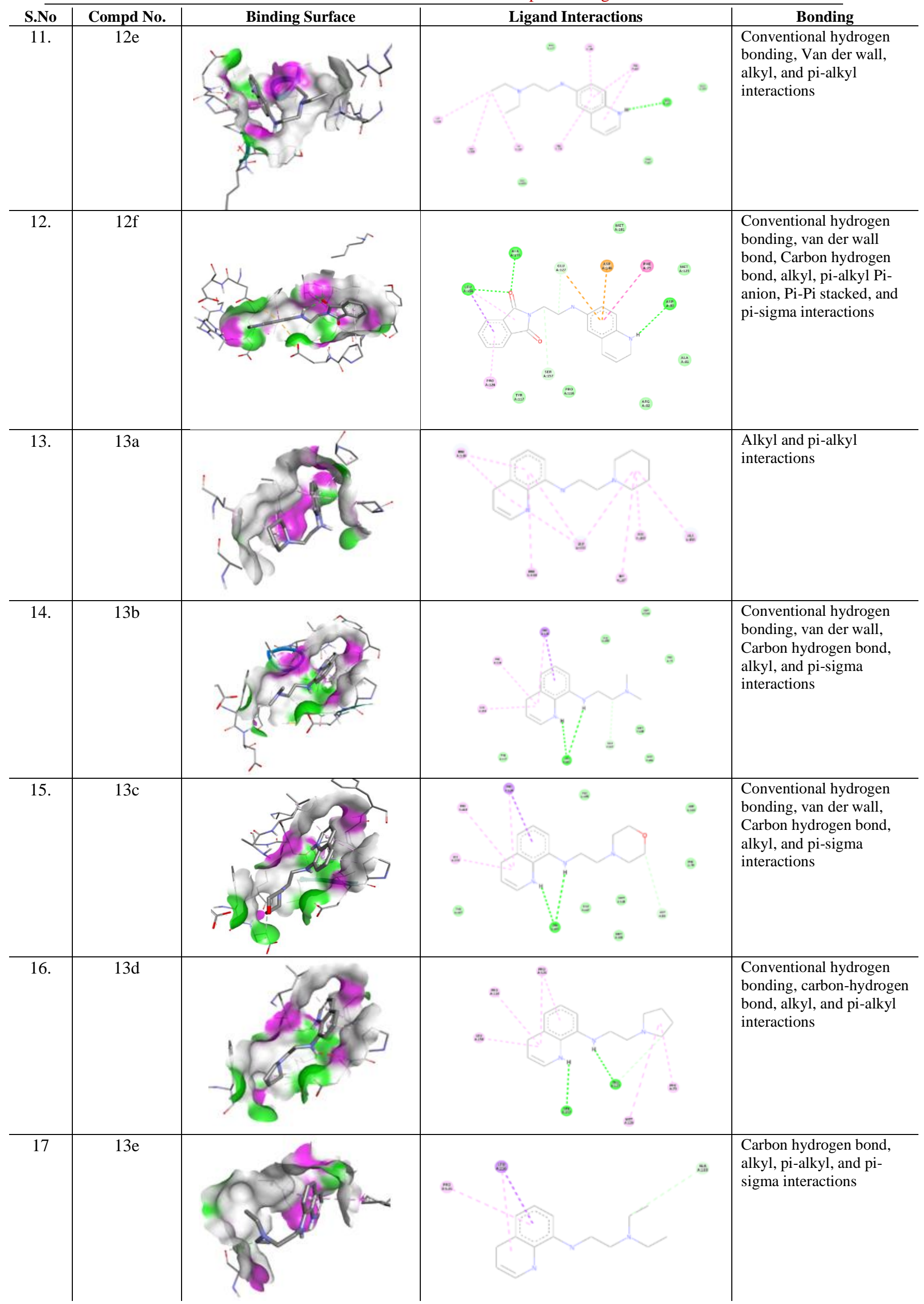




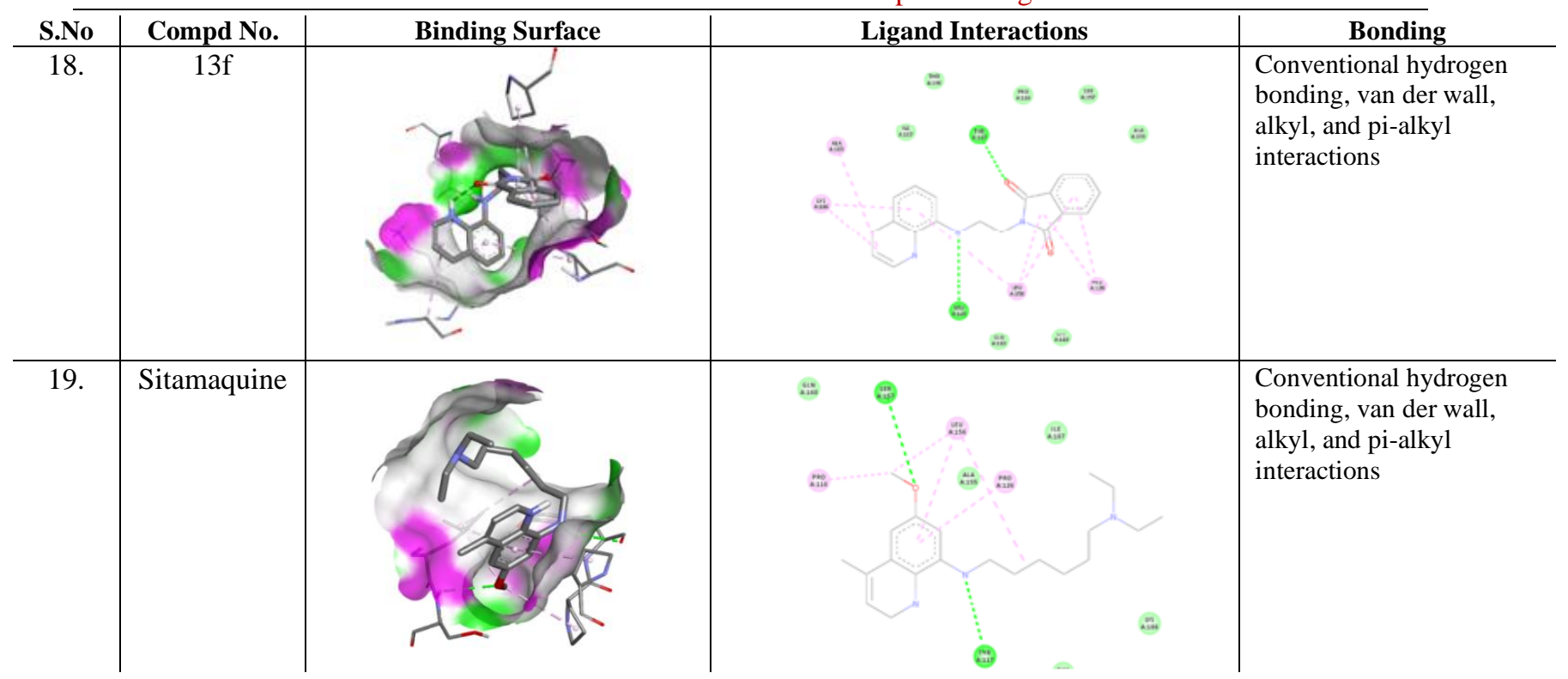

\subsection{Drug-likeness and ADMET analysis.}

Drug-likeness assessment and ADMET investigation for various compounds along with reference drugs are given in Tables 4 and 5, respectively. Inadequate pharmacokinetic and toxicity studies lead to failure of the drug development process in the final stages. Therefore, to escalate the drug discovery process, primary assessment of pharmacokinetics and toxicity studies is the mandate step.

The outcome of DruLiTo software demonstrates that all the synthesized compounds have admissible physicochemical properties such as hydrogen-bonding capacity, i.e., the numbers of hydrogen bond acceptors are under 10, and hydrogen bond donors are under 5, an important determinant of permeability. The molecular weight of the synthesized compounds is under 500, which predicts that these compounds can be easily transported, diffused, and absorbed compared to large molecules. LogP value, i.e., the octanol-water partition coefficient that usually quantified molecular lipophilicity is in the range of $0.087-1.562$, i.e., under 5 , which suggests the good permeability across the cell membrane and the polar surface area (TPSA), which is a good indicator of the bioavailability of the drug molecule is in the range of 27.63-61.77 and is well below the limits. All the values are in the acceptable range; hence all the synthesized drug molecules follow the Lipinski rule of five, as shown in Table 4.

Table 4. Drug-likeness assessment of various synthesized derivatives along with the standard drug.

\begin{tabular}{c|c|c|c|c|c|c|c} 
Compound No. & Mass & Log P & PSA & $\begin{array}{c}\text { Hydrogen } \\
\text { acceptor }\end{array}$ & $\begin{array}{c}\text { Hydrogen } \\
\text { donor }\end{array}$ & $\begin{array}{c}\text { Molar } \\
\text { refractivity }\end{array}$ & $\begin{array}{c}\text { No. of } \\
\text { rotatable } \\
\text { bonds }\end{array}$ \\
\hline 11a & 255.17 & 1.562 & 27.63 & 3 & 1 & 80.04 & 4 \\
\hline 11b & 215.14 & 0.698 & 27.63 & 3 & 1 & 73.28 & 4 \\
\hline 11c & 257.15 & 0.298 & 36.86 & 4 & 1 & 82.35 & 4 \\
\hline 11d & 241.16 & 1.204 & 27.63 & 3 & 1 & 77.13 & 4 \\
\hline 11e & 243.17 & 1.544 & 27.63 & 3 & 1 & 81.86 & 6 \\
\hline 11f & 317.12 & 0.821 & 61.77 & 5 & 1 & 99.88 & 4 \\
\hline 12a & 255.17 & 1.351 & 27.63 & 3 & 1 & 79.97 & 4 \\
\hline 12b & 215.14 & 0.487 & 27.63 & 3 & 1 & 73.20 & 4 \\
\hline 12c & 257.15 & 0.087 & 36.86 & 4 & 1 & 82.28 & 4 \\
\hline 12d & 241.16 & 0.993 & 27.63 & 3 & 1 & 77.06 & 4 \\
\hline 12e & 243.17 & 1.333 & 27.63 & 3 & 1 & 81.79 & 6 \\
\hline 12f & 317.12 & 0.610 & 61.77 & 5 & 1 & 99.81 & 4 \\
\hline 13a & 255.17 & 1.351 & 27.63 & 3 & 1 & 79.97 & 4 \\
\hline 13b & 215.14 & 0.487 & 27.63 & 3 & 1 & 73.20 & 4
\end{tabular}


https://doi.org/10.33263/BRIAC126.75037522

\begin{tabular}{c|c|c|c|c|c|c|c}
\hline Compound No. & Mass & Log P & PSA & $\begin{array}{c}\text { Hydrogen } \\
\text { acceptor }\end{array}$ & $\begin{array}{c}\text { Hydrogen } \\
\text { donor }\end{array}$ & $\begin{array}{c}\text { Molar } \\
\text { refractivity }\end{array}$ & $\begin{array}{c}\text { No. of } \\
\text { rotatable } \\
\text { bonds }\end{array}$ \\
\hline 13c & 257.15 & 0.087 & 36.86 & 4 & 1 & 82.28 & 4 \\
\hline 13d & 241.16 & 0.993 & 27.63 & 3 & 1 & 77.06 & 4 \\
\hline 13e & 243.17 & 1.333 & 27.63 & 3 & 1 & 81.79 & 6 \\
\hline 13f & 317.12 & 0.610 & 61.77 & 5 & 1 & 99.81 & 4 \\
\hline Sitamaquine & 343.26 & 3.242 & 24.50 & 4 & 1 & 94.38 & 11
\end{tabular}

All the synthetic derivatives displayed optimal Caco-2 permeability in a range of -4.442 to -4.693 . Since most of the drugs are administered through the oral route, so it is essential that the drug should be highly absorbed in intestinal tissue as all the compounds are HIA positive so they can be easily absorbed by the human intestine. The half-life (T1/2) values of various derivatives were in the range of 1.533 to 1.981 hours.

The median lethal dose $\left(\mathrm{LD}_{50}\right)$ is the dose amount of a tested molecule to kill $50 \%$ of the treated animals within a given period and, in the present, work the $\mathrm{LD}_{50}$ of various derivatives is in the comparable range with the reference since most of the synthesized compounds showed an acceptable range of ADMET profiles so they can be used efficiently as potent drug candidates (Table 5).

Table 5. ADMET analysis of various synthesized derivatives along with the standard drug.

\begin{tabular}{|c|c|c|c|c|c|c|c|}
\hline S. No. & Compound No. & $\log S$ & $\begin{array}{c}\text { Human } \\
\text { Intestinal } \\
\text { absorption }\end{array}$ & $\begin{array}{c}\text { Caco2 } \\
\text { Permeability }\end{array}$ & $\begin{array}{l}\text { Blood- } \\
\text { Brain } \\
\text { Barrier }\end{array}$ & $\begin{array}{c}\text { T } 1 / 2 \\
\text { (Half } \\
\text { Life } \\
\text { Time) }\end{array}$ & $\begin{array}{l}\text { LD50 } \\
\mathrm{mol} / \mathrm{kg}\end{array}$ \\
\hline 1. & 11a & -3.777 & ++ & -4.538 & +++ & 1.745 & 2.674 \\
\hline 2. & 11b & -2.691 & ++ & -4.462 & +++ & 1.533 & 2.728 \\
\hline 3. & $11 \mathrm{c}$ & -3.008 & ++ & -4.643 & +++ & 1.687 & 2.553 \\
\hline 4. & 11d & -3.294 & ++ & -4.509 & +++ & 1.646 & 2.674 \\
\hline 5. & 11e & -3.287 & ++ & -4.444 & +++ & 1.787 & 2.606 \\
\hline 6. & 11f & -4.455 & ++ & -4.691 & +++ & 1.981 & 2.601 \\
\hline 7. & 12a & -3.594 & ++ & -4.384 & +++ & 1.787 & 2.674 \\
\hline 8. & $12 \mathrm{~b}$ & -2.702 & ++ & -4.469 & +++ & 1.541 & 2.705 \\
\hline 9. & $12 \mathrm{c}$ & -3.037 & ++ & -4.643 & +++ & 1.719 & 2.561 \\
\hline 10. & 12d & -3.284 & ++ & -4.505 & +++ & 1.765 & 2.675 \\
\hline 11. & $12 \mathrm{e}$ & -3.278 & ++ & -4.442 & +++ & 1.847 & 2.609 \\
\hline 12. & $12 f$ & -4.466 & ++ & -4.693 & +++ & 1.962 & 2.605 \\
\hline 13. & 13a & -3.698 & ++ & -4.530 & +++ & 1.752 & 2.709 \\
\hline 14. & 13b & -2.686 & ++ & -4.463 & +++ & 1.567 & 2.746 \\
\hline 15. & $13 c$ & -2.975 & ++ & -4.624 & +++ & 1.699 & 2.551 \\
\hline 16. & 13d & -3.368 & ++ & -4.484 & +++ & 1.655 & 2.678 \\
\hline 17. & $13 e$ & -3.329 & ++ & -4.452 & +++ & 1.749 & 2.637 \\
\hline 18 & $13 f$ & -4.441 & ++ & -4.676 & +++ & 1.970 & 2.603 \\
\hline 19. & Sitamaquine & -5.124 & ++ & -4.664 & ++ & 2.213 & 2.538 \\
\hline
\end{tabular}

\section{Discussion}

In tropical countries, leishmaniasis is among one of the primary public problems leading to illness and death. Owing to the advent of widespread tolerance and toxicity of leishmaniasis, the available treatment opportunities are limited [43]. Quinoline scaffold is one of the widely used frameworks to synthesize novel derivatives with promising antiparasitic activity. The molecular structures of the newly synthesized quinoline derivatives were established based on spectral data. The infrared spectra of various synthesized compounds showed an absorption band at 3293-3709 $\mathrm{cm}^{-1}$ which confirms the presence of -NH functionality, the appearance of bands around $1450-1660 \mathrm{~cm}^{-1}$ and $1105-1271 \mathrm{~cm}^{-1}$ corresponding to $\mathrm{C}=\mathrm{C}$ aromatic stretching and $\mathrm{C}-\mathrm{N}$, respectively were also observed. Spectral data of all the compounds were consistent with the chemical structures. The triplet at $\delta 3-5 \mathrm{ppm}$ 
revealed the presence of the NH group. The multiplet at around $\delta 2.39-3.88 \mathrm{ppm}$ and a triplet at around $\delta 1.23-3.90 \mathrm{ppm}$ accounted for two methylene groups of the aliphatic chain.

Resistance of different strains of Leishmania species to conventional antileishmanial treatment has emerged as one of the world's most pressing issues. Thus, the development of new heterocyclic compounds having substantial biological and medicinal importance is a key objective of our study. Accordingly, various synthesized derivatives were screened for their invitro antileishmanial activity against the promastigote form of Leishmania donovani. The results of antileishmanial activity elucidated that most of the derivatives demonstrated good activity against promastigote form of Leishmania donovani with IC50 value ranging from 10.41 to $40.76 \mu \mathrm{g} / \mathrm{ml}$ as compared to standard sitamaquine with IC50 value of $10.09 \mu \mathrm{g} / \mathrm{ml}$. Amid the synthetic derivatives, the compounds 11f, $12 \mathrm{f}$ and $13 \mathrm{f}$ displayed comparable activity to standard with IC50 values of 13.61, 11.92, 10.41, whereas compounds 11a, 11c, 13a 13c exhibit moderate activity with IC50 values of $17.73,18.83,17.47$, and 17.87 . On the other hand, the rest of the compounds exhibit inferior activity as compared to the standard.

The outcome of DruLiTo software demonstrates that all the synthesized compounds have admissible physicochemical properties such as hydrogen-bonding capacity, i.e., the numbers of hydrogen bond acceptors are under 10, and hydrogen bond donors are under 5, an important determinant of permeability. The molecular weight of the synthesized compounds is under 500, which predicts that these compounds can be easily transported, diffused, and absorbed compared to large molecules. [ LogP value, i.e., the octanol-water partition coefficient that usually quantified molecular lipophilicity is in the range of 0.087-1.562, i.e., under 5, which suggests the good permeability across the cell membrane and the polar surface area (TPSA), which is a good indicator of the bioavailability of the drug molecule is in the range of 27.63-61.77 and is well below the limits. All the values are in the acceptable range; hence all the synthesized drug molecules follow the Lipinski rule of five [44-45].

All the synthesized compounds showed optimal Caco-2 permeability in a range of 4.442 to -4.693 . As most of the drugs are administered through the oral route, it is required that the drug be highly absorbed in intestinal tissue since all the compounds are HIA positive so the human intestine can easily absorb them, and all of them exhibit blood-brain-barrier crossing ability. The half-life (T1/2) values of various derivatives were in the range of 1.533 to 1.981 hours. The median lethal dose (LD50) usually represents the acute toxicity of compounds. It is the dose amount of a tested molecule to kill $50 \%$ of the treated animals within a given period. In the present work, the LD50 of various derivatives is in the comparable range with the reference. Since most synthesized compounds showed an acceptable range of ADMET profiles, they can be used efficiently as potent drug candidates.

\section{Conclusions}

The current investigation reports the synthesis, spectral characterization, the computational and antileishmanial activity of the newly synthesized series of quinoline derivatives, i.e., 11a to 13f. The results of antileishmanial activity reveal that 11f, 12f, and 13f were comparable in their action to standard drugs showing good human absorption and better $\log \mathrm{P}$ values, so it could be concluded that these compounds possessing quinoline moiety have significant antileishmanial activity and could lead to drug discovery of lead molecule for antileishmanial therapy. However, further thorough investigation such as in-vivo pharmacokinetics profiles is desired to appraise their potential to evolve into therapeutic agents. 


\section{Funding}

This research received no external funding.

\section{Acknowledgments}

The authors are thankful to Vice-chancellor Banasthali Vidyapith for providing the necessary research facilities. The corresponding author is also thankful to Amity University, Haryana.

\section{Conflicts of Interest}

The authors declare no conflict of interest.

\section{References}

1. Ganguly, S.; Das, N. K.; Barbhuiya, J. N.; Chatterjee, M. Post-kala-azar Dermal Leishmaniasis-an Overview. International journal of dermatology 2010, 49, 921-931, https://doi.org/10.1111/j.1365-4632.2010.04558.x.

2. Kumar, P.; Chatterjee, M.; Das, N. K. Post Kala-Azar Dermal Leishmaniasis: Clinical Features and Differential Diagnosis. Indian Journal of Dermatology 2021, 66, 24, http://doi:10.4103/ijd.IJD_602_20.

3. Gohil, C. J.; Kher, J. D. Review on Leishmaniasis. Biomedical Journal of Scientific \& Technical Research 2017, 1 (5), 1453-1455, https://doi.org/10.26717/BJSTR.2017.01.000460.

4. Haftom, M.; Petrucka, P.; Gemechu, K.; Nesro, J.; Amare, E.; Hailu, T.; Ashebir, Y.; Gebreheat, G.; Hagos, H.; Gebremedhin, D. Prevalence and Risk Factors of Human Leishmaniasis in Ethiopia: A Systematic Review and Meta-Analysis. Infectious Diseases and Therapy 2021, 10, 47-60, https://doi.org/10.1007/s40121-02000361-y.

5. Kasper, D.; Fauci, A.; Hauser, S.; Longo, D.; Jameson, J.; Loscalzo, J. Harrison's Principles of Internal Medicine, 19e; Mcgraw-hill New York, NY, USA:, 2015; 1 , https://accessmedicine. $m$ hmedical.com/content.aspx ?bookid=1130\&sectionid=79720773.

6. Kapil, S.; Singh, P. K.; Silakari, O. An Update on Small Molecule Strategies Targeting Leishmaniasis. European journal of medicinal chemistry 2018, 157, 339-367, https://doi.org/10.1016/j.ejmech.2018.08.012.

7. Steverding, D. The History of Leishmaniasis. Parasites \& vectors 2017, 10, 1-10, https://doi.org/10.1186/s13071-017-2028-5.

8. Glew, R. H.; Saha, A. K.; Das, S.; Remaley, A. T. Biochemistry of the Leishmania Species. Microbiological reviews 1988, 52, 412-432, http://doi.org/10.1128/mr.52.4.412-432.1988.

9. Boukthir, A.; Bettaieb, J.; Erber, A. C.; Bouguerra, H.; Mallekh, R.; Naouar, I.; Gharbi, A.; Alghamdi, M.; Plugge, E.; Olliaro, P. Psycho-Social Impacts, Experiences and Perspectives of Patients with Cutaneous Leishmaniasis Regarding Treatment Options and Case Management: An Exploratory Qualitative Study in Tunisia. Plos one 2020, 15, e0242494, https://doi.org/10.1371/journal.pone.0242494.

10. Bhattacharya, S. K.; Ganguly, S.; Sandipan Ganguly. Visceral Leishmaniasis. In Leishmaniases as Reemerging Diseases; Ghosal, A., Ed.; IntechOpen: Rijeka, 2018; https://doi.org/10.5772/intechopen.75907.

11. Singh, N.; Kumar, M.; Singh, R. K. Leishmaniasis: Current Status of Available Drugs and New Potential Drug Targets. Asian Pacific journal of tropical medicine 2012, 5, 485-497, https://doi.org/10.1016/S19957645(12)60084-4.

12. El-On, J.; Halevy, S.; Grunwald, M. H.; Weinrauch, L. Topical Treatment of Old World Cutaneous Leishmaniasis Caused by Leishmania Major: A Double-Blind Control Study. Journal of the American Academy of Dermatology 1992, 27, 227-231, https://doi.org/10.1016/0190-9622(92)70175-F.

13. Ruiz-Postigo, J. A.; Jain, S. Pentavalent Antimonials in the Treatment of Human Leishmaniasis. Antimony 2021, 303, https://doi.org/10.1515/9783110668711-013.

14. Ritmeijer, K.; Dejenie, A.; Assefa, Y.; Hundie, T. B.; Mesure, J.; Boots, G.; den Boer, M.; Davidson, R. N. A Comparison of Miltefosine and Sodium Stibogluconate for Treatment of Visceral Leishmaniasis in an Ethiopian Population with High Prevalence of HIV Infection. Clinical Infectious Diseases 2006, 43, $357-$ 364, https://doi.org/10.1086/505217.

15. Saha, A. K.; Mukherjee, T.; Bhaduri, A. Mechanism of Action of Amphotericin B on Leishmania Donovani Promastigotes. Molecular and biochemical parasitology 1986, 19, 195-200, https://doi.org/10.1016/01666851(86)90001-0. 
16. Chattopadhyay, A.; Jafurulla, M. A Novel Mechanism for an Old Drug: Amphotericin B in the Treatment of Visceral Leishmaniasis. Biochemical and biophysical research communications 2011, 416, 7-12, https://doi.org/10.1016/j.bbrc.2011.11.023.

17. Amlabu, W. E.; Antwi, C. A.; Awandare, G.; Gwira, T. M. Elucidating the Possible Mechanism of Action of Some Pathogen Box Compounds against Leishmania Donovani. PLoS neglected tropical diseases 2020, 14, e0008188, https://doi.org/10.1371/journal.pntd.0008188.

18. Pinto-Martinez, A. K.; Rodriguez-Durán, J.; Serrano-Martin, X.; Hernandez-Rodriguez, V.; Benaim, G. Mechanism of Action of Miltefosine on Leishmania Donovani Involves the Impairment of Acidocalcisome Function and the Activation of the Sphingosine-Dependent Plasma Membrane $\mathrm{Ca}^{2+}$ Channel. Antimicrobial agents and chemotherapy 2018, 62, e01614-17, https://doi.org/10.1128/AAC.01614-17.

19. Kaur, G.; Rajput, B. Comparative Analysis of the Omics Technologies Used to Study Antimonial, Amphotericin B, and Pentamidine Resistance in Leishmania. Journal of parasitology research 2014, https://doi.org/10.1155/2014/726328.

20. Ware, J. M.; O'Connell, E. M.; Brown, T.; Wetzler, L.; Talaat, K. R.; Nutman, T. B.; Nash, T. E. Efficacy and Tolerability of Miltefosine in the Treatment of Cutaneous Leishmaniasis. Clinical Infectious Diseases 2021, 73, e2457-e2562, https://doi.org/10.1093/cid/ciaa1238.

21. Malghani, Z.; Khan, A.-U.; Faheem, M.; Danish, M. Z.; Nadeem, H.; Ansari, S. F.; Maqbool, M. Molecular Docking, Antioxidant, Anticancer and Antileishmanial Effects of Newly Synthesized Quinoline Derivatives. Anti-Cancer Agents in Medicinal Chemistry (Formerly Current Medicinal Chemistry-Anti-Cancer Agents) 2020, 20, 1516-1529, https://doi.org/10.2174/1871520620666200516145117.

22. Coimbra, E. S.; Antinarelli, L. M. R.; Silva, N. P.; Souza, I. O.; Meinel, R. S.; Rocha, M. N.; Soares, R. P. P.; da Silva, A. D. Quinoline Derivatives: Synthesis, Leishmanicidal Activity and Involvement of Mitochondrial Oxidative Stress as Mechanism of Action. Chemico-biological interactions 2016, 260, 50-57, https://doi.org/10.1016/j.cbi.2016.10.017.

23. Yernale, G. A Comprehensive Review on the Biological Interest of Quinoline and Its Derivatives. Bioorganic \& Medicinal Chemistry 2021, 32, 115973, https://doi.org/10.1016/j.bmc.2020.115973.

24. Senerovic, L.; Opsenica, D.; Moric, I.; Aleksic, I.; Spasić, M.; Vasiljevic, B. Quinolines and Quinolones as Antibacterial, Antifungal, Anti-Virulence, Antiviral and Anti-Parasitic Agents. Advances in Microbiology, Infectious Diseases and Public Health 2019, 37-69, https://doi.org/10.1007/5584_2019_428.

25. da Gama, A. N. S.; Soeiro, M. N. C. Quinoline-Based Compounds as Key Candidates to Tackle Drug Discovery Programs of Microbicidal Agents. Current Pharmaceutical Design 2021, 27, 1757-1762, https://doi.org/10.2174/1381612826666201006125644.

26. Dib, M.; Ouchetto, H.; Ouchetto, K.; Hafid, A.; Khouili, M. Recent Developments of Quinoline Derivatives and Their Potential Biological Activities. Current Organic Synthesis 2021, 18, 248-269, https://doi.org/10.2174/1570179417666201216162055.

27. Tiwaria, S.; Kirarb, S.; Banerjeeb, U. C.; Babuc, N. K.; Singhc, S.; Singh, I. P. Synthesis and Biological Evaluation of Quinoline-Quinazolinones for Antimicrobial and Antileishmanial Potential. J. Indian Chem. Soc 2020, 97, 1251-1258.

28. Razzaghi-Asl, N.; Sepehri, S.; Ebadi, A.; Karami, P.; Nejatkhah, N.; Johari-Ahar, M. Insights into the Current Status of Privileged N-Heterocycles as Antileishmanial Agents. Molecular diversity 2020, 24, 525-569, https://doi.org/10.1007/s11030-019-09953-4.

29. Ashutosh; Gupta, S.; Ramesh; Sundar, S.; Goyal, N. Use of Leishmania Donovani Field Isolates Expressing the Luciferase Reporter Gene in in Vitro Drug Screening. Antimicrobial agents and chemotherapy 2005, 49, 3776-3783, https://doi.org/10.1128/AAC.49.9.3776-3783.2005.

30. Zeleke, D.; Eswaramoorthy, R.; Belay, Z.; Melaku, Y. Synthesis and Antibacterial, Antioxidant, and Molecular Docking Analysis of Some Novel Quinoline Derivatives. Journal of Chemistry 2020, https://doi.org/10.1155/2020/1324096.

31. Phillips, C. L.; Ullman, B.; Brennan, R. G.; Hill, C. P. Crystal Structures of Adenine Phosphoribosyltransferase from Leishmania Donovani. The EMBO Journal 1999, 18, 3533-3545, https://doi.org/10.1093/emboj/18.13.3533.

32. Discovery Studio Modeling Environment, Release 2017. BIOVIA, Dassault Systèmes, San Diego.

33. Laskowski, R. A.; Moss, D. S.; Thornton, J. M. Main-Chain Bond Lengths and Bond Angles in Protein Structures. Journal of molecular biology 1993, 231, 1049-1067, https://doi.org/10.1107/S0021889892009944. 
34. Laskowski, R. A.; Rullmann, J. A. C.; MacArthur, M. W.; Kaptein, R.; Thornton, J. M. AQUA and PROCHECK-NMR: Programs for Checking the Quality of Protein Structures Solved by NMR. Journal of biomolecular NMR 1996, 8, 477-486, https://doi.org/10.1007/BF00228148.

35. Trott, O.; Olson, A. J. AutoDock Vina: Improving the Speed and Accuracy of Docking with a New Scoring Function, Efficient Optimization, and Multithreading. Journal of computational chemistry 2010, 31, 455461, https://doi.org/10.1002/jcc.21334.

36. Yamashita, F.; Hashida, M. In Silico Approaches for Predicting ADME Properties of Drugs. Drug metabolism and pharmacokinetics 2004, 19, 327-338, https://doi.org/10.2133/dmpk.19.327.

37. Mälkiä, A.; Murtomäki, L.; Urtti, A.; Kontturi, K. Drug Permeation in Biomembranes: In Vitro and in Silico Prediction and Influence of Physicochemical Properties. European Journal of Pharmaceutical Sciences 2004, 23, 13-47, https://doi.org/10.1016/j.ejps.2004.05.009.

38. van de Waterbeemd, H.; Gifford, E. ADMET in Silico Modelling: Towards Prediction Paradise? Nature reviews Drug discovery 2003, 2, 192-204, https://doi.org/10.1038/nrd1032.

39. Bickerton, G. R.; Paolini, G. v; Besnard, J.; Muresan, S.; Hopkins, A. L. Quantifying the Chemical Beauty of Drugs. Nature chemistry 2012, 4, 90-98, https://doi.org/10.1038/nchem.1243.

40. Kar, S.; Leszczynski, J. Open Access in Silico Tools to Predict the ADMET Profiling of Drug Candidates. Expert Opinion on Drug Discovery 2020, 15, 1473-1487, https://doi.org/10.1080/17460441.2020.1798926.

41. Lipinski, C. A.; Lombardo, F.; Dominy, B. W.; Feeney, P. J. Experimental and Computational Approaches to Estimate Solubility and Permeability in Drug Discovery and Development Settings. Advanced drug delivery reviews 1997, 23, 3-25, https://doi.org/10.1016/S0169-409X(96)00423-1.

42. Dong, J.; Wang, N.-N.; Yao, Z.-J.; Zhang, L.; Cheng, Y.; Ouyang, D.; Lu, A.-P.; Cao, D.-S. ADMETlab: A Platform for Systematic ADMET Evaluation Based on a Comprehensively Collected ADMET Database. Journal of cheminformatics 2018, 10 (1), 1-11, https://doi.org/10.1186/s13321-018-0283-x.

43. Horácio, E. C. A.; Hickson, J.; Murta, S. M. F.; Ruiz, J. C.; Nahum, L. A. Perspectives from Systems Biology to Improve Knowledge of Leishmania Drug Resistance. Frontiers in Cellular and Infection Microbiology 2021, 11, 351, https://doi.org/10.3389/fcimb.2021.653670.

44. Mahdian, S.; Ebrahim-Habibi, A.; Zarrabi, M. Drug Repurposing Using Computational Methods to Identify Therapeutic Options for COVID-19. Journal of Diabetes \& Metabolic Disorders 2020, 19, 691-699, https://doi.org/10.1007/s40200-020-00546-9.

45. Rajkhowa, S.; Jha, A. N.; Deka, R. C. Anti-Tubercular Drug Development: Computational Strategies to Identify Potential Compounds. Journal of Molecular Graphics and Modelling 2015, 62, 56-68, https://doi.org/10.1016/j.jmgm.2015.09.007. 